\title{
The promise and peril of chemical probe negative controls
}

\author{
Jinyoung Lee ${ }^{\#}$, Matthieu Schapira ${ }^{\#, \&}$
}

\footnotetext{
¥ Structural Genomics Consortium, University of Toronto, Toronto, Ontario M5G 1L7, Canada

! Department of Pharmacology \& Toxicology, University of Toronto, Toronto, Ontario M5S 1A8, Canada

*To whom correspondence should be addressed: matthieu.schapira@utoronto.ca
}

\section{METHODS}

\section{Assembling a set of matched protein pairs}

To assemble a valid collection of pairs of protein structures in complex with identical ligands, the following conditions were used: (1) co-crystalized ligands must meet Lipinski rule of five; (2) co-crystalized ligands must be occupying a welldefined pocket rather than laying on a flat surface: ligand desolvation value $>70 \%$. These two conditions were addressed as described in our previous work ${ }^{1}$. (3) Structures must have a resolution of $3 \AA$ or better. (4) Finally, ligands must be cocrystalized with at least two structurally unrelated proteins. To meet this condition, we first kept the structure with the highest resolution when multiple structures of the same protein (defined by its InterPro ID) and the same ligand (defined by its PDB ID) were available. Next, for each pair of protein in complex with the same ligand, we used ICM to calculate backbone RMSD between protein domains occupied by the ligand (defined by aminoacids within $10 \AA ̊$ of the bound ligand).

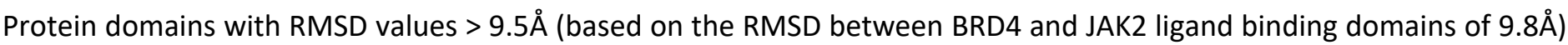
were judged structurally dissimilar and retained.

\section{Energy calculation}

The energy of the co-crystalized ligand and the energies of the methylated analogues were calculated for each structure. All calculations were conducted with ICM (Molsoft, San Diego). First, the co-crystallized ligand was tethered to its original binding pose (tzWeight $=1$ ) and minimized locally in the internal coordinates space with default energy terms (including Van der Waals (VdW), hydrogen bonds, electrostatics and torsion terms). The Van de Waals energy of the bound ligand was then calculated with the command "show ey" followed by "Energy("vw,14")" using a soft Van der Waals energy term $($ vwMethod $=3)$. Soft Van der Waals eliminates small steric clashes that could be accommodated by a small adjustment of the ligand binding pose. The overall energy calculation process was repeated three times for each structure, and the averages of those three rounds were used for the analysis. Standard deviations were low and are reported in Supp Table 3. If the Van der Waals energy of the un-methylated, energy-minimized ligand was positive, the structure was rejected.

Next, the Van der Waals energy of methylated analogues were calculated as follows. The co-crystallized ligand in its energy-minimized pose was mono-methylated in silico with ICM and tethered to the original, unmodified ligand with a high tether weight (tzWeight $=200$.) to constrain the conformation of the virtual negative control to the binding pose of the un-methylated crystallized ligand (previously relaxed as described above). The energy of the tethered virtual negative 
control was globally minimized using ICM's Monte Carlo method (command: montecarlo), followed by a local minimization (command: minimize). The Van der Waals energy of the methylated compound was then calculated as described above. This process of generating and calculating energy of a virtual negative control was repeated for each hydrogen atom of the parent compound. A hydrogen atom was judged as sterically restrained if replacing it with a methyl group resulted in positive Van der Waals energy of the bound ligand. 


\begin{tabular}{|c|c|c|c|c|c|c|}
\hline $\begin{array}{l}\text { Protein } \\
\text { Family }\end{array}$ & $\begin{array}{c}\text { Specific } \\
\text { Target }\end{array}$ & Probe & $\begin{array}{c}\text { Negative } \\
\text { Control (NC) }\end{array}$ & $\begin{array}{l}\text { Probe Off- } \\
\text { Target }\end{array}$ & $\begin{array}{c}\text { NC Off- } \\
\text { Target }\end{array}$ & $\begin{array}{c}\text { \% Off- } \\
\text { Target } \\
\text { Lost }\end{array}$ \\
\hline \multirow{6}{*}{$\begin{array}{l}\text { Methyltran } \\
\text { sferase }\end{array}$} & \multirow{6}{*}{$\begin{array}{c}\text { SUV420 } \\
\text { H1/H2 }\end{array}$} & & \multirow{6}{*}{ A-197 } & $\begin{array}{c}\text { A1 [Ki } 0.021 \\
\mu \mathrm{M}]\end{array}$ & $\begin{array}{c}\mathrm{A} 1[\mathrm{Ki} 3.1 \\
\mu \mathrm{M}]\end{array}$ & \multirow{6}{*}{83} \\
\hline & & & & $\begin{array}{c}\text { A2A }[\mathrm{Ki} 0.028 \\
\mu \mathrm{M}]\end{array}$ & $\begin{array}{c}\text { A2A [Ki } 20 \\
\mu \mathrm{M}]\end{array}$ & \\
\hline & & & & $\mathrm{A} 3[\mathrm{Ki} 2 \mu \mathrm{M}]$ & $\begin{array}{c}\mathrm{A} 3[\mathrm{Ki} 13 \\
\mu \mathrm{M}]\end{array}$ & \\
\hline & & & & $\begin{array}{c}\text { Cl- channel } \\
\text { (GABA-gated) } \\
{[\mathrm{Ki} 1.8 \mu \mathrm{M}]}\end{array}$ & $\mathrm{n} / \mathrm{a}$ & \\
\hline & & & & $\begin{array}{c}\text { NK2 [Ki } 4.1 \\
\mu \mathrm{M}]\end{array}$ & $\mathrm{n} / \mathrm{a}$ & \\
\hline & & & & $\begin{array}{c}\text { delta } 2 \text { (DOP) } \\
{[\mathrm{Ki} 9 \mu \mathrm{M}]}\end{array}$ & $\mathrm{n} / \mathrm{a}$ & \\
\hline \multirow{6}{*}{ YEATS } & \multirow{6}{*}{$\begin{array}{l}\text { MMT1, } \\
\text { MLLT3 }\end{array}$} & \multirow{6}{*}{ NV } & \multirow{6}{*}{ NVS-MLLT-C } & $\begin{array}{c}\text { PIM1 [IC } 50.6 \\
\mu \mathrm{M}]\end{array}$ & $\mathrm{n} / \mathrm{a}$ & \multirow{6}{*}{83} \\
\hline & & & & $\begin{array}{c}\text { TRB2 [IC } 503.5 \\
\mu \mathrm{M}]\end{array}$ & $\begin{array}{c}\text { TRB2 }\left[\mathrm{IC}_{50}\right. \\
3.5 \mu \mathrm{M}]\end{array}$ & \\
\hline & & & & $\begin{array}{c}\text { ACES [IC50 } \\
0.25 \mu \mathrm{M}]\end{array}$ & $\mathrm{n} / \mathrm{a}$ & \\
\hline & & & & $\begin{array}{c}\mathrm{H} 3\left[\mathrm{IC}_{50} 0.29\right. \\
\mu \mathrm{M}]\end{array}$ & $\mathrm{n} / \mathrm{a}$ & \\
\hline & & & & 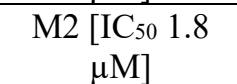 & $\mathrm{n} / \mathrm{a}$ & \\
\hline & & & & $\begin{array}{c}\text { NET }\left[\mathrm{IC}_{50} 8.7\right. \\
\mu \mathrm{M}]\end{array}$ & $\mathrm{n} / \mathrm{a}$ & \\
\hline \multirow{5}{*}{$\begin{array}{l}\text { Bromodo } \\
\text { mains }\end{array}$} & \multirow{5}{*}{$\begin{array}{l}\text { BPTF } \\
\text { (FALZ) }\end{array}$} & \multirow{5}{*}{ NVS-BPTF-1 } & \multirow{5}{*}{ NVS-BPTF-C } & $\begin{array}{c}\mathrm{Ad} 3\left[\mathrm{IC}_{50} 4.9\right. \\
\mu \mathrm{M}]\end{array}$ & $\begin{array}{c}\mathrm{Ad} 3\left[\mathrm{IC}_{50} 13\right. \\
\mu \mathrm{M}]\end{array}$ & \multirow{5}{*}{0} \\
\hline & & & & $\begin{array}{c}\text { D3 }\left[\mathrm{IC}_{50} 3.5\right. \\
\mu \mathrm{M}]\end{array}$ & $\begin{array}{c}\mathrm{D} 3\left[\mathrm{IC}_{50} 0.95\right. \\
\mu \mathrm{M}]\end{array}$ & \\
\hline & & & & $\begin{array}{c}\mathrm{H} 3\left[\mathrm{IC}_{50} 4.0\right. \\
\mu \mathrm{M}]\end{array}$ & $\begin{array}{c}\mathrm{H} 3\left[\mathrm{IC}_{50} 23\right. \\
\mu \mathrm{M}]\end{array}$ & \\
\hline & & & & $\begin{array}{c}\text { ACES [IC } 5030 \\
\mu \mathrm{M}]\end{array}$ & $\begin{array}{c}\text { ACES [IC } 50 \\
5.5 \mu \mathrm{M}]\end{array}$ & \\
\hline & & & & $\mathrm{n} / \mathrm{a}$ & $\begin{array}{c}\mathrm{COX}_{2}\left[\mathrm{IC}_{50}\right. \\
0.8 \mu \mathrm{M}]\end{array}$ & \\
\hline \multirow{11}{*}{$\begin{array}{l}\text { Methyltran } \\
\text { sferase }\end{array}$} & \multirow{11}{*}{ SMYD2 } & \multirow{11}{*}{$\begin{array}{c}\text { LLY-507 } \\
\text { (Multiple Off- } \\
\text { target Effects) }\end{array}$} & \multirow{11}{*}{ SGC705 } & $\begin{array}{c}\text { 5-HT1A [Ki } \\
0.12 \mu \mathrm{M}]\end{array}$ & $\begin{array}{c}\text { 5-HT1A [IC } 50 \\
0.14 \mu \mathrm{M}]\end{array}$ & \multirow{11}{*}{45} \\
\hline & & & & $\begin{array}{c}\text { 5-HT1B [Ki } 1.2 \\
\mu \mathrm{M}]\end{array}$ & $\mathrm{n} / \mathrm{a}$ & \\
\hline & & & & $\mathrm{n} / \mathrm{a}$ & $\begin{array}{c}\text { 5-HT2A [IC } 50 \\
0.07 \mu \mathrm{M}]\end{array}$ & \\
\hline & & & & $\begin{array}{c}\text { 5-HT2B [Ki } 2.2 \\
\mu \mathrm{M}]\end{array}$ & $\begin{array}{c}\text { 5-HT2B [IC } 50 \\
1.4 \mu \mathrm{M}]\end{array}$ & \\
\hline & & & & $\begin{array}{c}\text { 5-HT2C }[\mathrm{Ki} 1.9 \\
\mu \mathrm{M}]\end{array}$ & $\begin{array}{c}\text { 5-HT2C }\left[\mathrm{IC}_{50}\right. \\
1.5 \mu \mathrm{M}]\end{array}$ & \\
\hline & & & & $\begin{array}{c}\text { 5-HT5a [Ki } 1.9 \\
\mu \mathrm{M}]\end{array}$ & $\mathrm{n} / \mathrm{a}$ & \\
\hline & & & & $\begin{array}{c}\text { 5-HT6 [Ki } 1.4 \\
\mu \mathrm{M}]\end{array}$ & $\begin{array}{c}\text { 5-HT6 [Ki } 2.2 \\
\mu \mathrm{M}] \\
\end{array}$ & \\
\hline & & & & $\begin{array}{c}\text { 5-HT7 [Ki } 0.3 \\
\mu \mathrm{M}]\end{array}$ & $\begin{array}{c}\text { 5-HT7 [Ki } 2.5 \\
\mu \mathrm{M}]\end{array}$ & \\
\hline & & & & $\begin{array}{c}\text { Alpha1A [Ki } \\
0.24 \mu \mathrm{M}]\end{array}$ & $\begin{array}{c}\text { Alpha1A [Ki } \\
0.82 \mu \mathrm{M}]\end{array}$ & \\
\hline & & & & $\begin{array}{c}\text { Alpha1B [Ki } \\
0.91 \mu \mathrm{M}]\end{array}$ & $\begin{array}{c}\text { Alpha1B [Ki } \\
3.5 \mu \mathrm{M}]\end{array}$ & \\
\hline & & & & $\begin{array}{c}\text { Alpha2A [Ki } \\
0.66 \mu \mathrm{M}]\end{array}$ & $\begin{array}{c}\text { Alpha2A [Ki } \\
0.52 \mu \mathrm{M}]\end{array}$ & \\
\hline
\end{tabular}




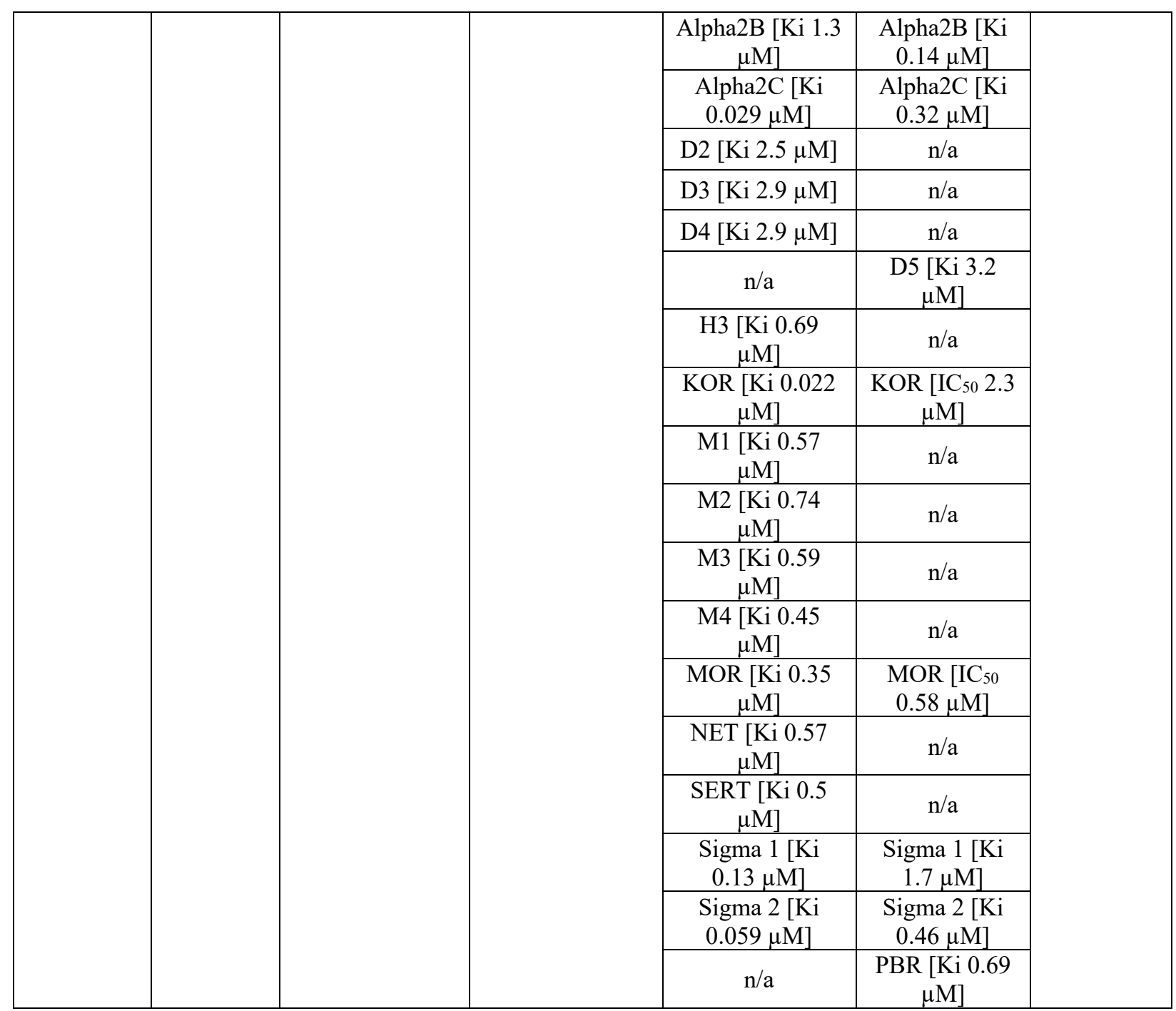

Supplementary Table 1: Profiling data for four chemical probes and their negative controls. Only proteins where compounds are active are shown. NVS-BPTF-1 and its negative control NVS-BPTF-C were profiled on a panel from the Novartis Institute of Biomedical Research consisting of 15 GPCRs, 3 transporters, 3 nuclear hormone receptors, 48 kinases and 7 enzymes ${ }^{2}$. NVS-MLLT- 1 and its negative control NVS-MLLT-C were profiled against 59 kinases, 7 additional enzymes, 16 GPCRs and 5 other diverse proteins ${ }^{3}$. A-196 and A-197 were profiled against 54 GPCRS and transporters from CEREP 4 . LLY-507 and SGC-705 were profiled against of 45 GPCRs and transporters from the NIMH Psychoactive Drug Screening Program 5 . 


\begin{tabular}{|c|c|c|c|c|c|c|c|}
\hline \multicolumn{3}{|c|}{ Chemical probe } & \multicolumn{3}{|c|}{ Methylated analogue } & \multirow[b]{2}{*}{$\begin{array}{l}\text { Methylated } \\
\text { analog is } \\
\text { active }\end{array}$} & \multirow{2}{*}{$\begin{array}{c}\text { Methylated } \\
\text { analog is } \\
\text { predicted } \\
\text { active } \\
\end{array}$} \\
\hline Structure & $\begin{array}{l}\mathrm{IC}_{50} \\
(\mathrm{nM})\end{array}$ & $\begin{array}{c}\text { VdW } \\
\text { energy } \\
\text { (kcal/mol) } \\
\end{array}$ & Structure & $\begin{array}{c}\mathrm{IC}_{50} \\
(\mathrm{nM})\end{array}$ & $\begin{array}{c}\text { VdW } \\
\text { energy } \\
\text { (kcal/mol) }\end{array}$ & & \\
\hline $\begin{array}{c}\text { FM-381 } \\
\text { Compound } 4 \\
\text { (PDB: 5lwm) }\end{array}$ & 0.127 & $-8.327 \pm 0$ & $\begin{array}{l}\text { Compound } 5 \\
\text { (PDB: 5lwn) }\end{array}$ & 0.154 & $\begin{array}{c}-5.915 \pm \\
0.02\end{array}$ & yes & yes \\
\hline $\begin{array}{c}\text { FM-381 } \\
\text { Compound } 4 \\
\text { (PDB: 5lwm) }\end{array}$ & 0.127 & $-8.327 \pm 0$ & & $>1,000$ & $\begin{array}{c}0.981 \pm \\
0.00\end{array}$ & no & no \\
\hline $\begin{array}{c}\text { BAY-707 } \\
\text { (PDB: 5nhy) }\end{array}$ & 2.3 & $-9.428 \pm 0$ & BA) & $>20,000$ & $\begin{array}{c}9.649 \pm \\
0.03\end{array}$ & no & no \\
\hline $\begin{array}{c}\text { CPI-1612 } \\
\text { Compound } 17 \\
\text { (PDB: 6v8n) }\end{array}$ & 8 & $-15.39 \pm 0$ & $\begin{array}{l}\text { Compound } 12 \\
\text { (PDB: 6v90) }\end{array}$ & 19 & $\begin{array}{c}-9.585 \pm \\
0.8\end{array}$ & yes & yes \\
\hline
\end{tabular}

Supplementary Table 2: Experimentally measured and computationally predicted effects of methylating a chemical probe. Compound structures, experimental $\mathrm{IC}_{50}$ values and computationally calculated Van der Waals clashes for the bound ligand (VdW energy) are shown for the JAK3 kinase ligands FM-381, compound 5 and FM-479,7, the NUDT1 ligands BAY-707 and BAY-604 ${ }^{8}$ and the EP300 ligands CPI-1612 and compounds $12^{9}$.

Supplementary Table 3 is provided as a separate excel file 


\begin{tabular}{|c|c|c|}
\hline $\begin{array}{l}\text { Cluster } \text { (NLigand, }_{\text {PPoB) }} \text {; } \\
\text { Example Ligand }\end{array}$ & Example PDB $_{1}$ & Example $\mathrm{PDB}_{2}$ \\
\hline $1_{(1,2) ; 08 j}$ & Bromodomain; BRD4; 3u5k; 50\% & Cytochrome P450; CP3A4; 5te8; 33\% \\
\hline $2_{(1,2)} ; 1 \mathrm{~m} 3$ & Protein kinase domain; JAK2; 4ji9;60\% & Bromodomain; BRD4; 4076;75\% \\
\hline $3(2$, & Protein kinase domain; PLK1; 2rku; 43\% & Bromodomain; BRD4; 4ogi; $60 \%$ \\
\hline $4_{(1,2)} ; 1 \mathrm{qk}$ & Protein kinase domain; $\mathrm{CDK} 2 ; 4 \mathrm{kd} 1 ; 47 \%$ & Bromodomain; BRD4; 4070;64\% \\
\hline $5(1,4)$; imn & Glutathione S-transferase; PGES2; 1z9h;0\% & Serum albumin, N-terminal; ALBU; 2bxk; N/A \\
\hline
\end{tabular}




\begin{tabular}{|c|c|c|}
\hline $6_{(1,2) ; p 1 b}$ & Nuclear hormone receptor, ligand-binding domain; & Amine oxidase; AOFB; 4a79; 100\% \\
\hline $7_{(1,2) ; \mathrm{vgh}}$ & Protein kinase domain; ALK; 2yfx; 33\% & NUDIX hydrolase domain; 8ODP; $4 \mathrm{c} 9 \mathrm{w} ; 14 \%$ \\
\hline $8_{(1,2)} ; \mathrm{p} 6 \mathrm{u}$ & Peptidase family A1 domain; BACE1;5t1u;67\% & Cytochrome P450; CP2D6; 5tft; 40\% \\
\hline $9_{(1,2)}$; si5 & Peptidase family A1 domain; BACE1;4xxs; $60 \%$ & Cytochrome P450; CP2D6; 4xry; 33\% \\
\hline $10_{(6,19) ;}$ adn & Aminoacyl-tRJA synthase, class II (G/P/S/T); & METK2; 6fcd; $80 \%$ \\
\hline $11_{(1,2) ;} ; \mathrm{a} 1 \mathrm{z}$ & Cupin-like domain 8; HIF1N; 5opc; 67\% & $\begin{array}{l}\text { Oxoglutarate/iron-dependent dioxygenase; } \\
\text { EGLN1; 50x6;50\% }\end{array}$ \\
\hline
\end{tabular}




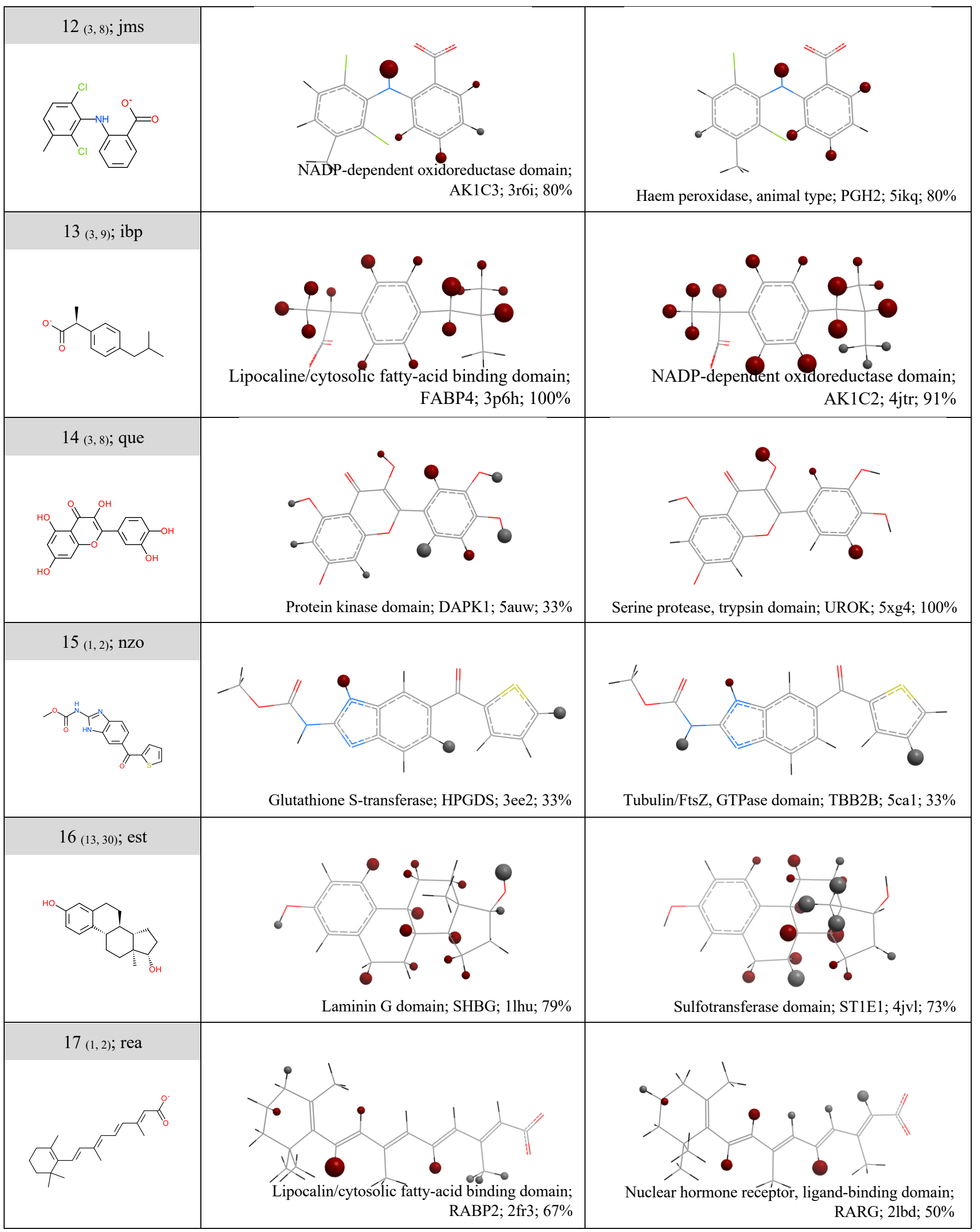


Supplementary Table 4: pcR across 17 chemical clusters. Representative compounds are shown for the 17 chemical clusters composed of molecules each found in two or more structures in the PDB in complex with unrelated protein domains. For each cluster, the number of compounds and number of proteins is shown in parenthesis, followed by the PDB ligand name of the representative compound (first column). For each chemical cluster, a representative compound is shown and constrained hydrogens are highlighted as in Figure 2 when bound to two unrelated proteins. The percent risk $(\mathrm{pcR})$ that the negative control of a chemical probe binding to one protein do not bind to the other protein is shown. The full list of 41 compounds found in 90 protein pairs is available in supplementary Table 3. Clustering was conducted with ICM, using a Tanimoto fingerprint similarity cut-off of 0.5 to make sure that each cluster is chemically distinct. 


\begin{tabular}{|c|c|c|c|c|c|c|c|c|c|c|c|c|c|c|c|c|c|}
\hline \multirow{2}{*}{$\begin{array}{c}\text { Domain Pair } \\
\text { (Domain 1; Domain 2) }\end{array}$} & \multicolumn{17}{|c|}{ Ligand Cluster } \\
\hline & 1 & 2 & 3 & 4 & 5 & 6 & 7 & 8 & 9 & 10 & 11 & 12 & 13 & 14 & 15 & 16 & 17 \\
\hline $\begin{array}{l}\text { NUDIX hydrolase domain; Protein kinase } \\
\text { domain }\end{array}$ & & & & & & & & & & & & & & & & & \\
\hline $\begin{array}{l}\text { G protein-coupled receptor, rhodopsin-like } \\
\text { Protein kinase domain }\end{array}$ & & & & & & & & & & & & & & & & & \\
\hline $\begin{array}{l}\text { G protein-coupled receptor, rhodopsin-like } \\
\text { Histidine triad (HIT) protein }\end{array}$ & & & & & & & & & & & & & & & & & \\
\hline $\begin{array}{l}\text { G protein-coupled receptor, rhodopsin-like } \\
\text { Aminoacyl-tRNA synthetase, class II (G/ P/ } \\
S / T)\end{array}$ & & & & & & & & & & & & & & & & & \\
\hline $\begin{array}{l}\text { G protein-coupled receptor, rhodopsin-like } \\
\text { Histidine kinase/HSP90-like ATPase }\end{array}$ & & & & & & & & & & & & & & & & & \\
\hline $\begin{array}{l}\text { G protein-coupled receptor, rhodopsin-like } \\
\text { S-adenosyl-L-homocysteine hydrolase, NAD } \\
\text { binding domain }\end{array}$ & & & & & & & & & & & & & & & & & \\
\hline $\begin{array}{l}\text { G protein-coupled receptor, rhodopsin-like } \\
\text { S-adenosylmethionine synthetase, central } \\
\text { domain }\end{array}$ & & & & & & & & & & & & & & & & & \\
\hline $\begin{array}{l}\text { G protein-coupled receptor, rhodopsin-like } \\
\text { Histone-lysine N-methyltransferase DOT1 } \\
\text { domain }\end{array}$ & & & & & & & & & & & & & & & & & \\
\hline $\begin{array}{l}\text { Nuclear hormone receptor, ligand-binding } \\
\text { domain; Lipocalin/cytosolic fatty-acid bindi } \\
\text { domain }\end{array}$ & & & & & & & & & & & & & & & & & \\
\hline $\begin{array}{l}\text { Nuclear hormone receptor, ligand-binding } \\
\text { domain; Oxysterol-binding protein }\end{array}$ & & & & & & & & & & & & & & & & & \\
\hline $\begin{array}{l}\text { Nuclear hormone receptor, ligand-binding } \\
\text { domain; Protein kinase domain }\end{array}$ & & & & & & & & & & & & & & & & & \\
\hline $\begin{array}{l}\text { Nuclear hormone receptor, ligand-binding } \\
\text { domain; Sulfotransferase domain }\end{array}$ & & & & & & & & & & & & & & & & & \\
\hline $\begin{array}{l}\text { Nuclear hormone receptor, ligand-binding } \\
\text { domain; Cytochrome P450 }\end{array}$ & & & & & & & & & & & & & & & & & \\
\hline $\begin{array}{l}\text { Nuclear hormone receptor, ligand-binding } \\
\text { domain; Laminin } G \text { domain }\end{array}$ & & & & & & & & & & & & & & & & & \\
\hline $\begin{array}{l}\text { Nuclear hormone receptor, ligand-binding } \\
\text { domain; Amine oxidase }\end{array}$ & & & & & & & & & & & & & & & & & \\
\hline $\begin{array}{l}\text { Nuclear hormone receptor, ligand-binding } \\
\text { domain; MD-2-related lipid-recognition } \\
\text { domain }\end{array}$ & & & & & & & & & & & & & & & & & \\
\hline $\begin{array}{l}\text { Nuclear hormone receptor, ligand-binding } \\
\text { domain; Glutathione S-transferase }\end{array}$ & & & & & & & & & & & & & & & & & \\
\hline $\begin{array}{l}\text { Nuclear hormone receptor, ligand-binding } \\
\text { domain; Serum albumin, N-terminal }\end{array}$ & & & & & & & & & & & & & & & & & \\
\hline $\begin{array}{l}\text { Nuclear hormone receptor, ligand-binding } \\
\text { domain; NADP-dependent oxidoreductase } \\
\text { domain }\end{array}$ & & & & & & & & & & & & & & & & & \\
\hline $\begin{array}{l}\text { Nuclear hormone receptor, ligand-binding } \\
\text { domain; Serpin domain }\end{array}$ & & & & & & & & & & & & & & & & & \\
\hline $\begin{array}{l}\text { Nuclear hormone receptor, ligand-binding } \\
\text { domain; Niemann-Pick } \mathrm{C} 1, \mathrm{~N} \text {-terminal }\end{array}$ & & & & & & & & & & & & & & & & & \\
\hline
\end{tabular}




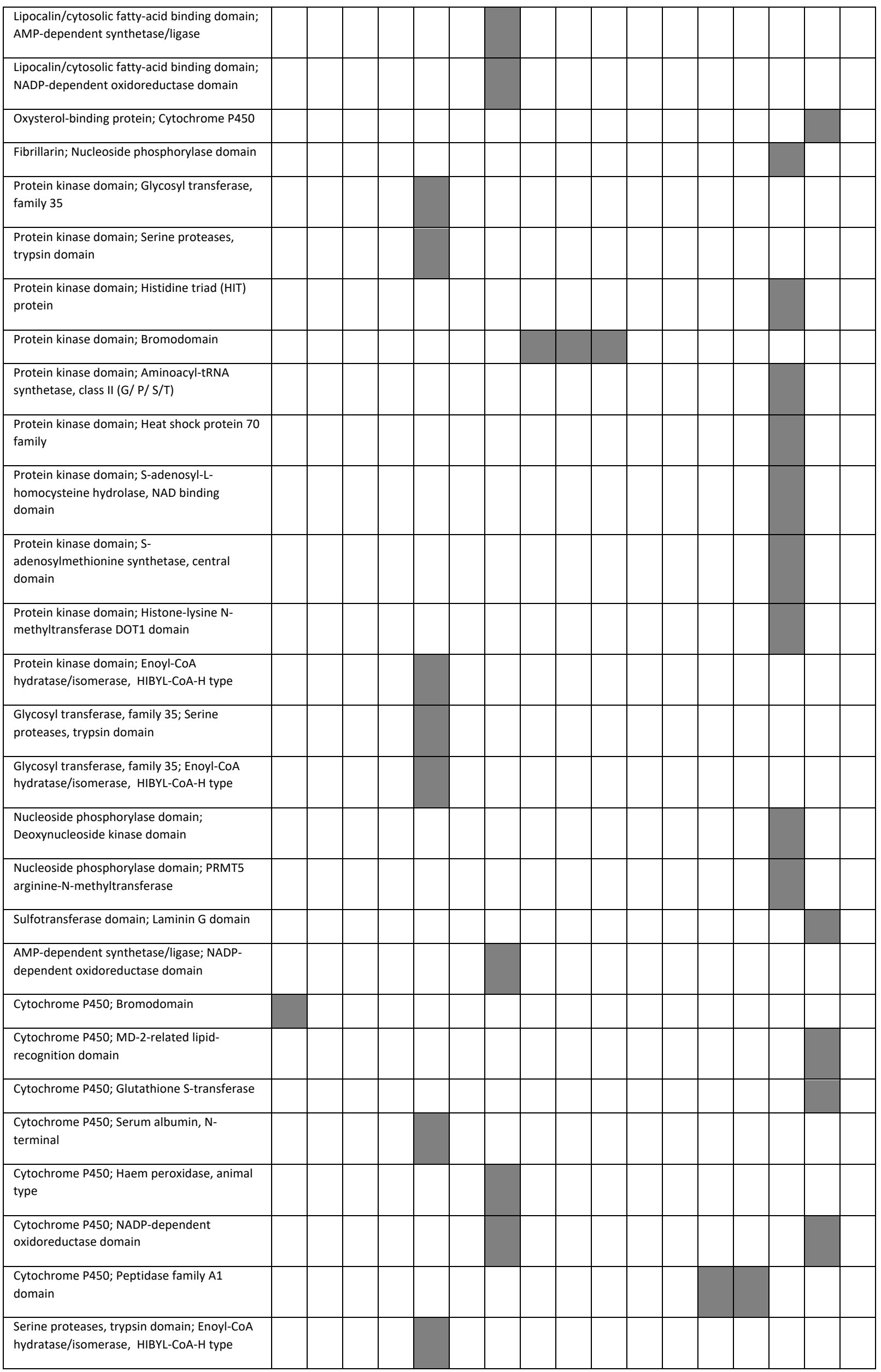




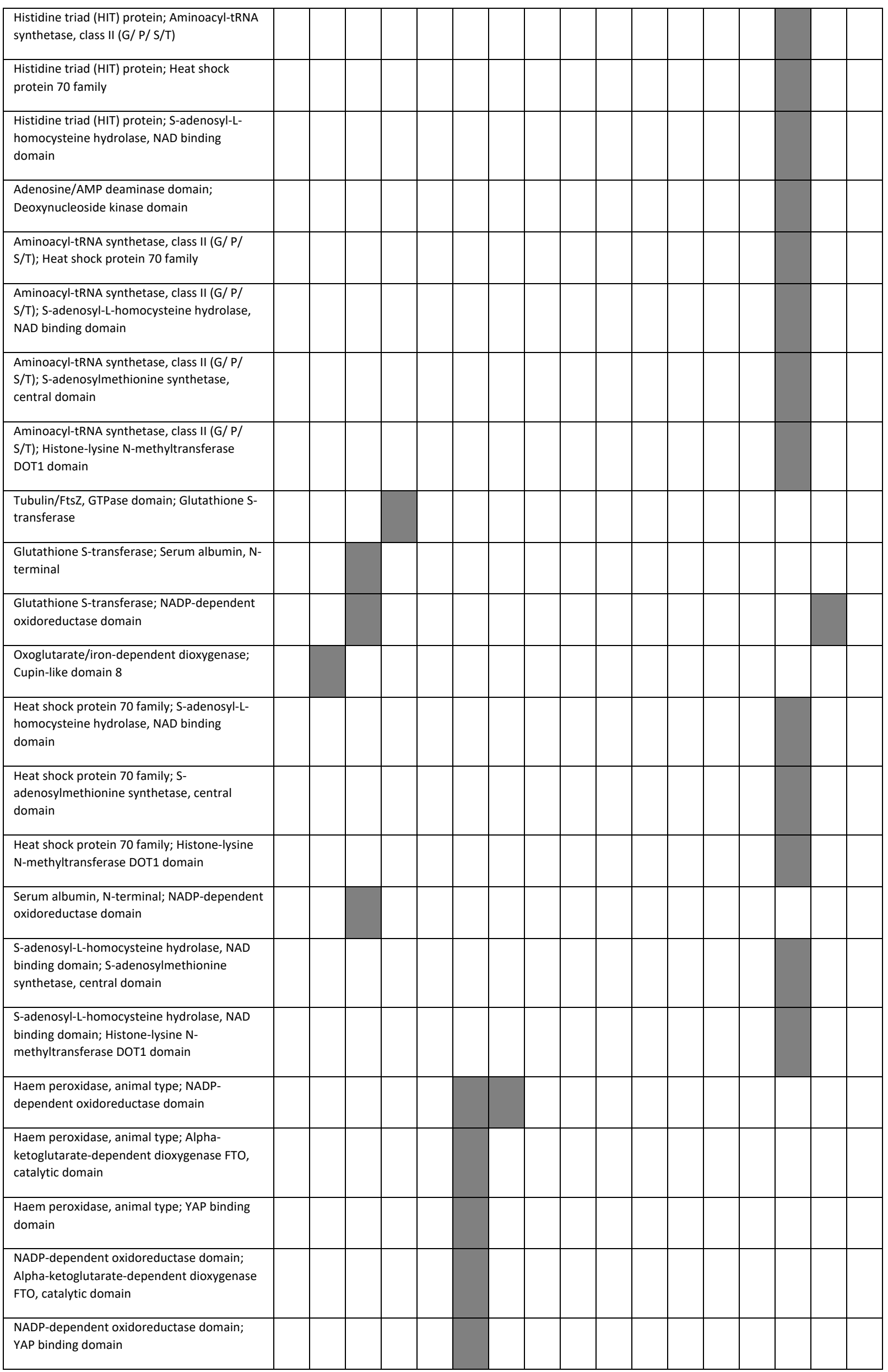


Supplementary Table 5: For each pair of unrelated protein domains, chemical clusters where at least one ligand is found in complex with both domains in the PDB are highlighted.
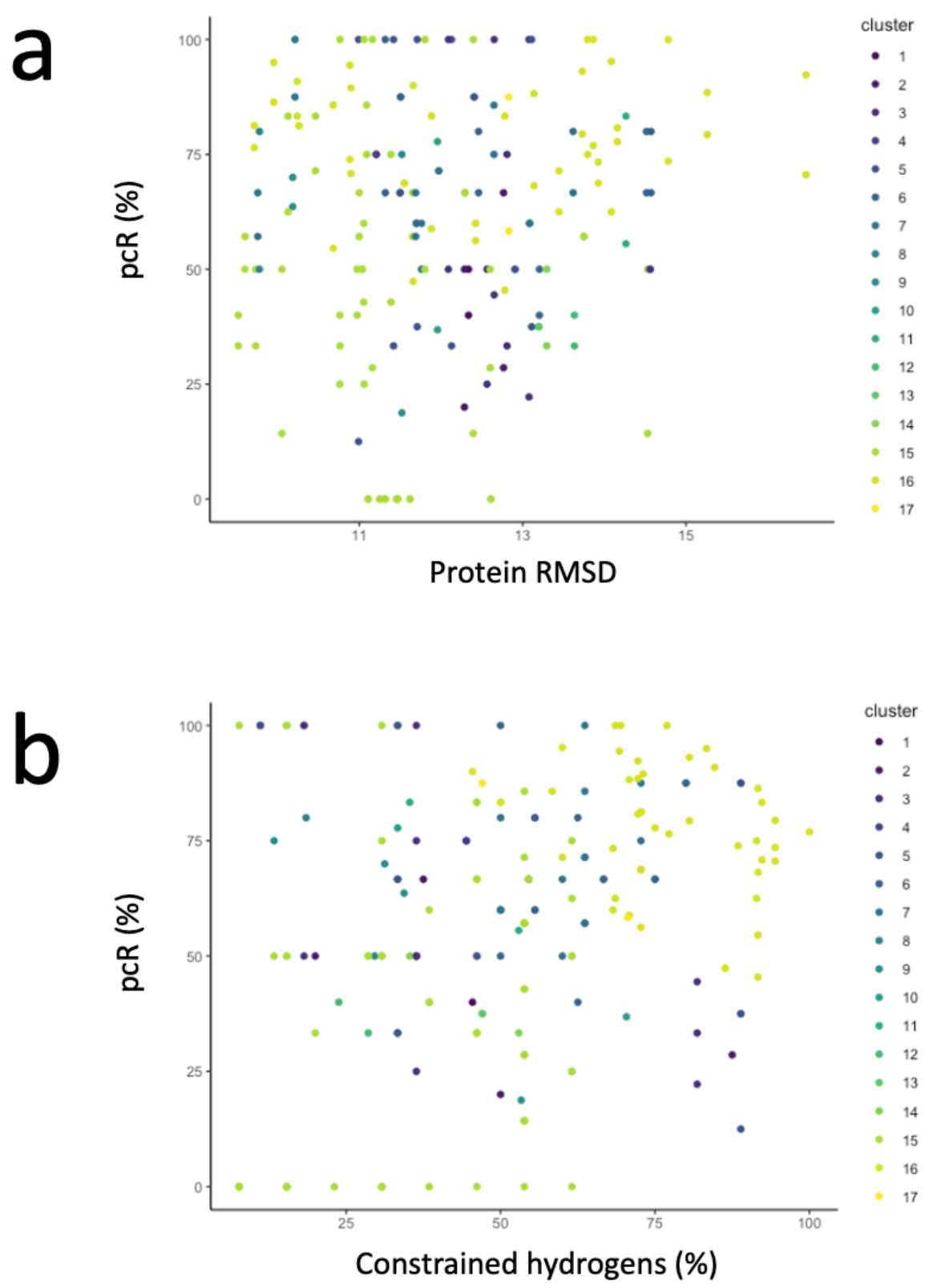

Supplementary Figure 1: pcR values do not correlate with the structural similarity between proteins domains (a) or the percentage of hydrogens constrained in a binding site (b).

\section{Computer Codes}

All codes are shown below and can be run with ICM-Pro 3.8-7d (Molsoft, San Diego) available from molsoft.com.

1- Data Filter

errorAction $=3$

\# Purpose: To filter the "drug-like" ligand data based on following criteria:

\# $\quad$ 1) desolvaiton value

\# 2) different domains - identified based on different InterPro IDs

\# Input: all_ip_with_pdb (drug_like ligands last updated Dec 17, 2019), pdb_resolu (table) 
\#---

\# INPUT VARIABLES

$t=$ all_ip_with_pdb

\#-

\#-

function FilterlP(s)

\# Prerequisite - this function needs table "pdb_resolu" and table "t" prepared

\# Purpose - takes in ligand_name "s" as an argument and returns table "t_final" if and only if the

\# $\quad \mathrm{s}$ is co-crystalized with more than one domain (identified using InterPro ID comparisons)

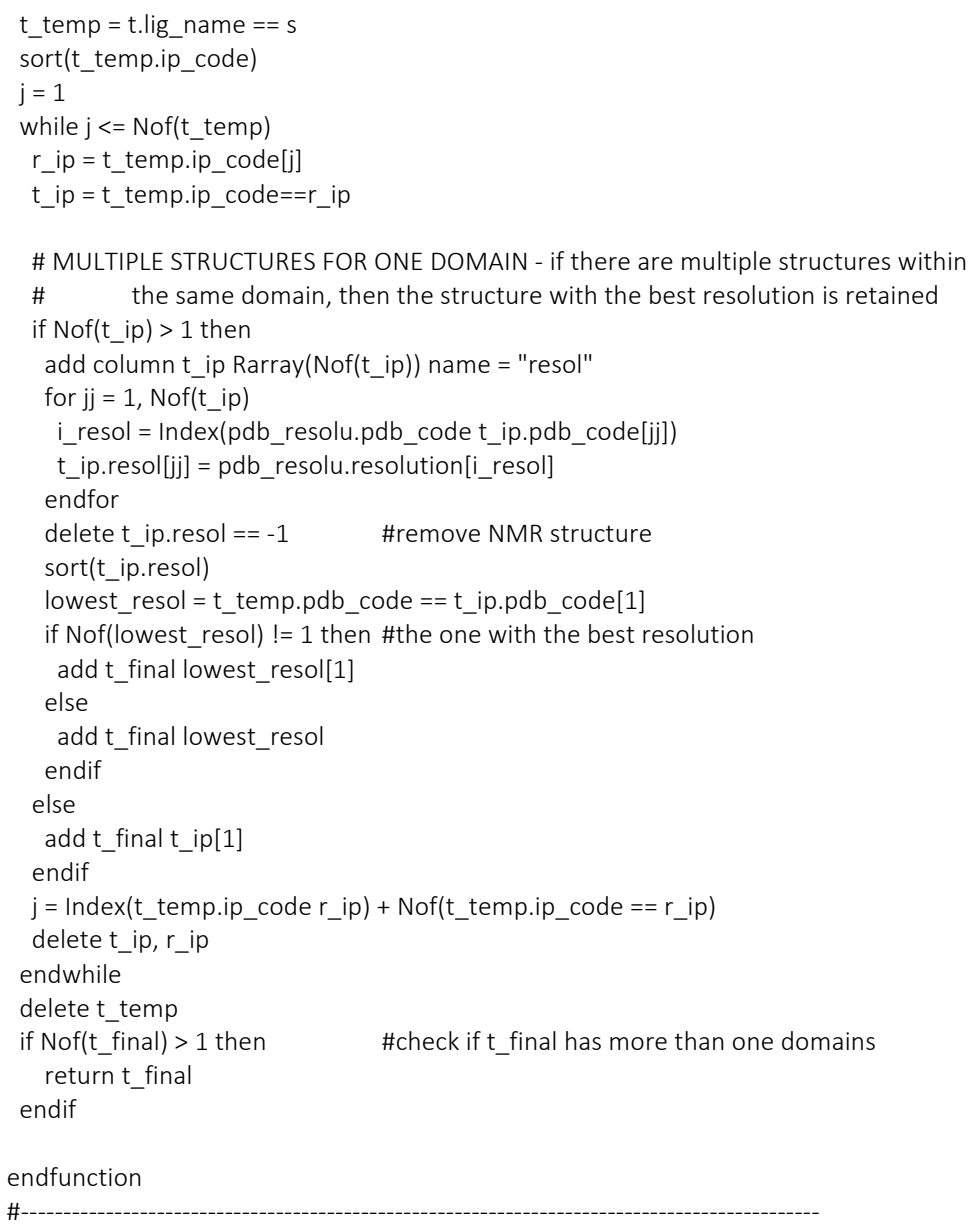

\section{\# MAIN SCRIPT STARTS:}

\# STEP 0 - preparing the table

\# Note - STEP 0 handles exceptions that are manually implemented for the initial data used in my analysis.

\# If you are using the same initial table as my analysis, keep these exceptions.

\# If not, please check them to ensure they are applicable for your analysis as well.

\# You can add or remove any of the exceptions in STEP 0 based on the data you are using.

t.protein_chain = Tolower(t.protein_chain) \# protein_chain must be in a lower case

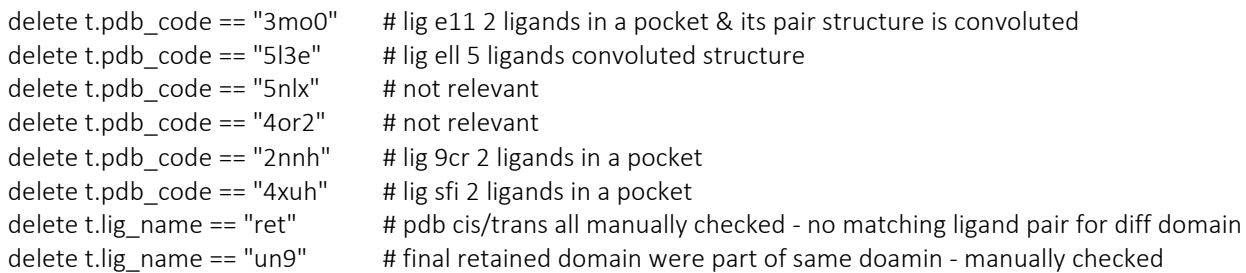

\# STEP 1 - remove desolvation value below 70.

delete t.ligand_desolvation $<70$.

\# STEP 2 - compare domains to ensure each "ligand" has more than one distinct InterProlD 
$\mathrm{i}=1$

while $\mathrm{i}<=\operatorname{Nof}(\mathrm{t})$

r_lig = t.lig_name[i]

num_lig = Nof(t.lig_name $\left.==r \_l i g\right)$

if num_lig $>1$ then

filterip = FilterIP(r_lig)

if Type(filterip) != "integer" then

add lig_ips filterip

endif

delete filterip

endif

$\mathrm{i}=\mathrm{i}+$ num_lig

delete r_lig

endwhile

$\mathrm{t}=$ lig_ips

delete lig_ips

\# STEP 3 - remove duplicate PDB structures since some PDB 3D proteins structures are assigned to multiple InterProlD

$\mathrm{i}=1$

while $\mathrm{i}<=$ Nof(t)

r_lig = t.lig_name[i]

t_temp $=$ t.lig_name $==r_{-}$lig

$i=i+\operatorname{Nof}\left(t \_t e m p\right)$

$\mathrm{a}=1$

while $\mathrm{a}<=$ Nof(t_temp)

sort(t_temp.pdb_code)

r_pdb = t_temp.pdb_code[a]

if Nof(t_temp.pdb_code $==$ r_pdb) ! 1 then

delete t_temp[a]

endif

$a=$ Index(t_temp.pdb_code r_pdb) +1

delete r_pdb

endwhile

if Nof(t_temp) $>1$ then

add lig_ips t_temp

endif

delete r_lig, t_temp

endwhile

delete $\mathrm{t}$

2- ICM conversion

errorAction $=3$

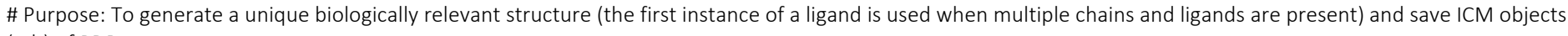
(.ob) of PDB structures

\# Input: lig_ips (table)

\# Output: N/A (ICM objects saved in the folder specified by the variable "save_location")

\# Prerequisite: set directory to the variable "pdb_special_cases_location" and "save_location"

\#--

\# INPUT VARIABLES

$\mathrm{t}=$ lig_ips

\# Input - directory: ensure that your directory to a specific folder ends with "\I"

pdb_special_cases_location = "'" \# directory where edited PDB files are saved

save_location = "'" \# directory where ICM objects will be saved

\#--

\#-

function TrimStructure( S)

\# Purpose - to prepare ICM objects of PDB 3D protein structures for further analysis

\# ARGUMENT:

\# S[1] is pdb code

\# S[2] is lig_name (with protein_chain added)

\# S[3] is protein_chain

\# STEP 1 - read PDB structure 


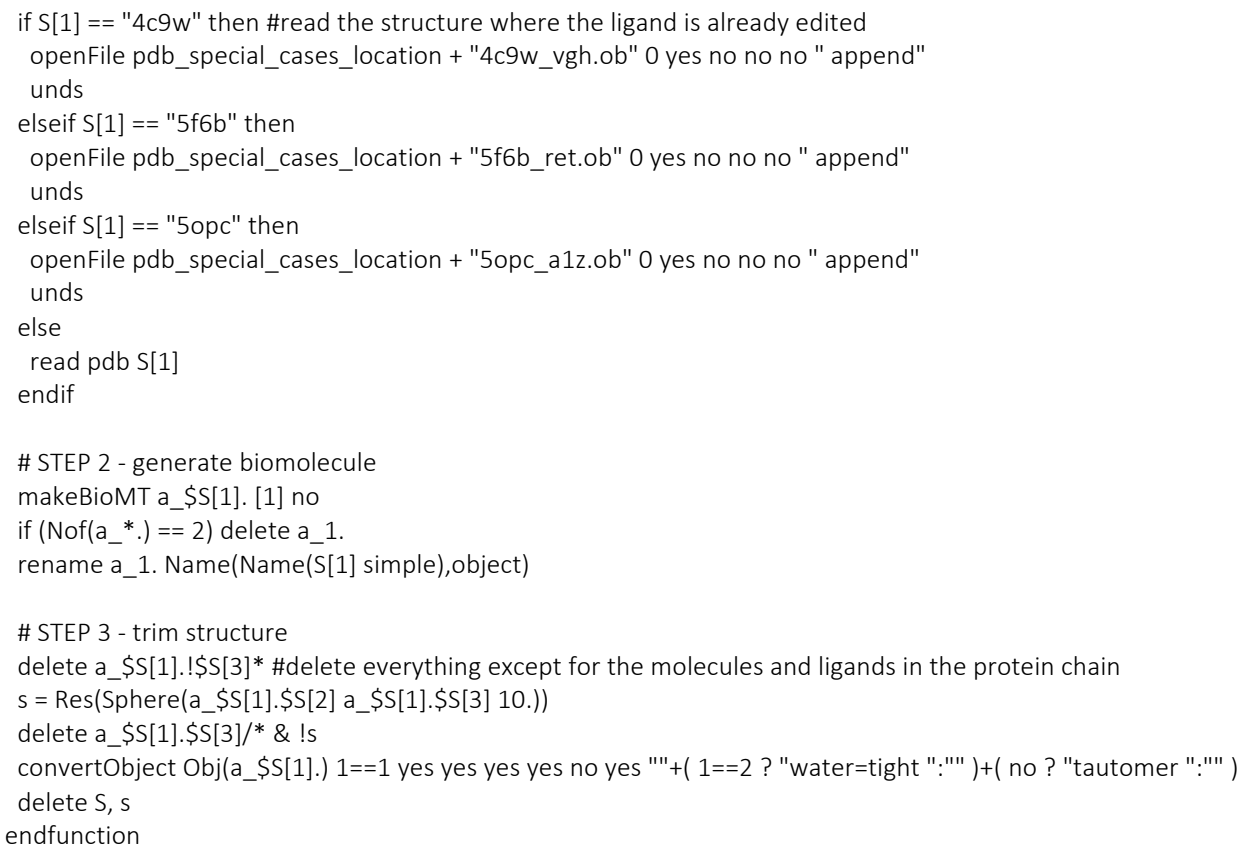




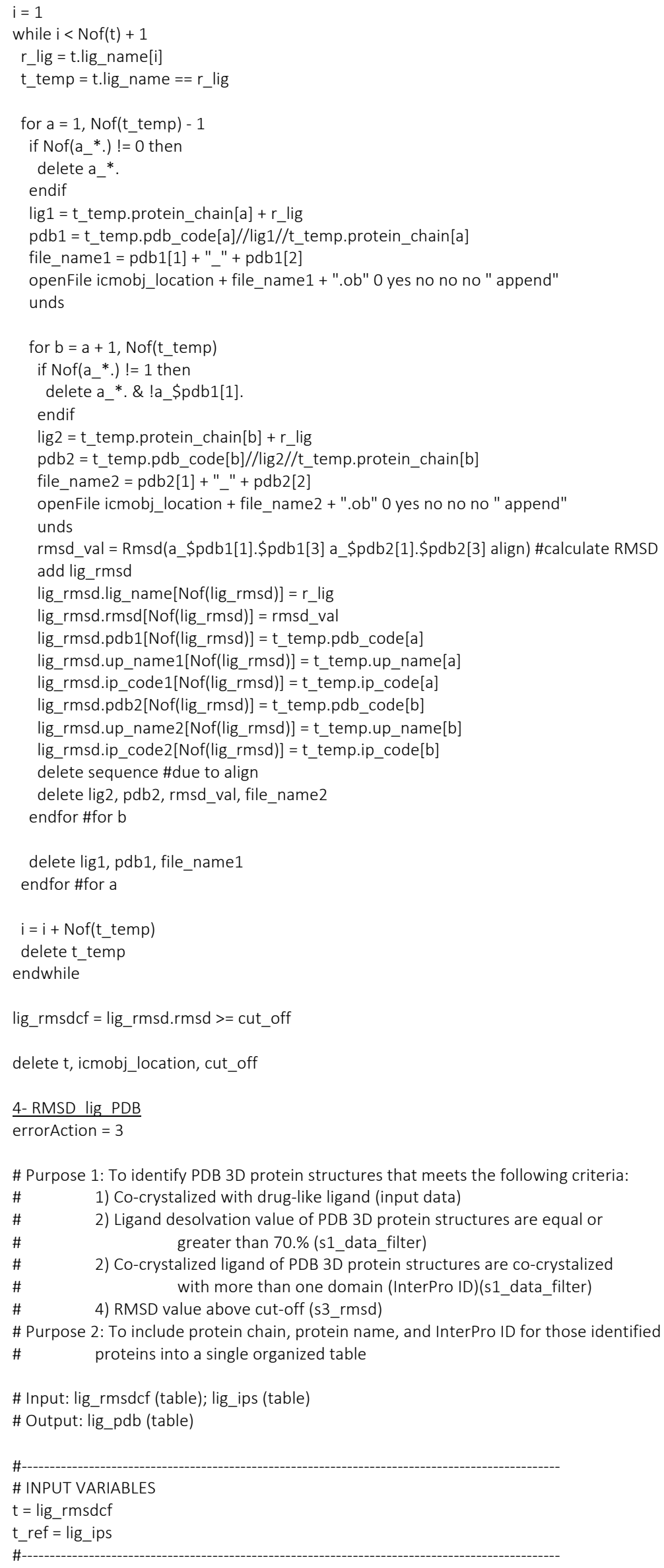




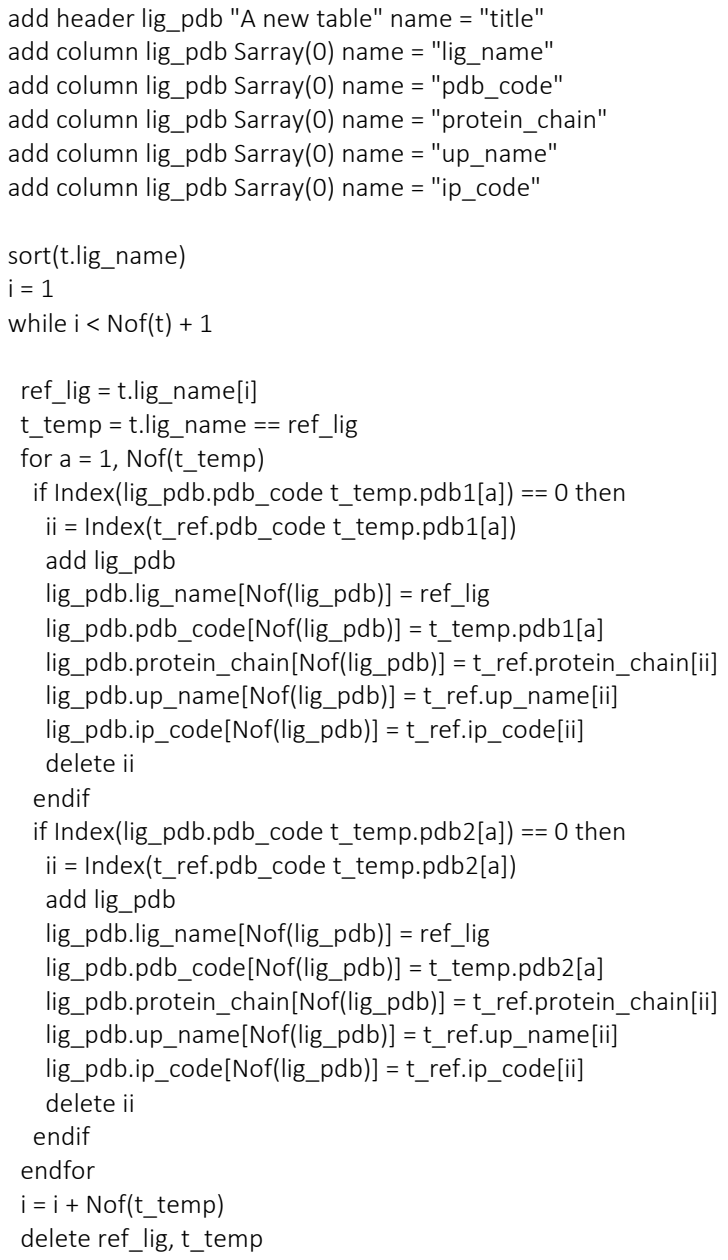

endwhile

delete $t$, t_ref

5- Lig-H

errorAction $=3$

\# Purpose: Generate a template table for the ligand for each pdb_code, where hydrogen atoms of the ligands \# are identified, organized, and saved into a table (tt)

\# Input: lig_pdb (table)

\# Output: lig_h (table)

\# Prerequisite: set directory to the variable "icmobj_location" and "save_location"

$\#-$

\# INPUT VARIABLE

$t=$ lig_pdb

\# Input - directory: ensure that your directory to a specific folder ends with "\\" icmobj_location = "'" \# use save_location specified in the script s2_bicm_conversion save_location = "' $\quad$ \# directory where template table "tt" will be saved

\#-

\# MAIN SCRIPT STARTS:

for $i=1, \operatorname{Nof}(t)$

add header tt "A new table" name = "title" add column tt $\operatorname{Sarray}(0)$ name = "lig_name" add column tt Sarray $(0)$ name $=$ "pdb code" add column tt Sarray(0) name = "lig_h" add column tt Sarray(0) name = "bound_atom"

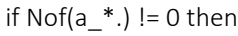
delete $a_{-}{ }^{*}$. 
endif

$\mathrm{cpdb}=\mathrm{t}$. pdb_code[i]//t.protein_chain[i]+t.lig_name[i]//t.protein_chain[i]

file_name $=c p d b[1]+"{ }^{-}+\operatorname{cpdb}[2]$

openFile icmobj_location + file_name + ".ob" 0 yes no no no " append" \#TEST

unds

\#STEP 1 - make a table for all hydrogen atoms

lig_h $=$ Name $\left(a_{\_} \$ c p d b[1] . \$ c p d b[2] / / h^{*}\right) \#$ \#identify hydrogen atoms of the ligand

for $a=1$, Nof(lig_h)

add tt

tt.lig_name[Nof(tt)] = t.lig_name[i]

tt.pdb_code[Nof(tt)] = cpdb[1]

tt.lig_h[Nof(tt)] = lig_h[a]

bound_atom = Name(Next(a_\$cpdb[1].\$cpdb[2]//\$lig_h[a] bond) \& !a_\$cpdb[1].\$cpdb[2]//vt*)

sort(bound_atom) \#necessary because of "vt"

tt.bound_atom[Nof(tt)] = bound_atom[1]

delete bound_atom

endfor

\#STEP 2 - identify if they are hydrogen atoms of a methyl group

add column tt Sarray(Nof(tt)) name = "methyl"

$a=1$

while a $<$ Nof $(\mathrm{tt})+1$

ref_atom $=$ tt.bound_atom[a]

t_temp $=$ tt.bound_atom $==$ ref_atom

if $\operatorname{Nof}(\mathrm{t}$ temp) $==3$ then

tt.methyl[a] = "yes"

tt.methyl $[a+1]=$ "yes"

tt.methyl[a+2] = "yes"

endif

$\mathrm{a}=\mathrm{a}+\operatorname{Nof}\left(\mathrm{t} \_\right.$temp $)$

delete t_temp

endwhile

\#STEP 3 - save the final table tt to lig_energy/lig_ $h$

write binary tt save_location + file_name + ".icb"

delete cpdb, file_name, lig_h, tt

endfor

delete icmobj_location, save_location, t

6-Energy Calc

errorAction $=3$

default_terms = "vw,14,hb,el,to,ss" \# default energy terms

\# Purpose: To calculate the initial energies of the ligands and energies of computationally methylated ligands co-crystalized with 3D protein structures "round" number of times.

\# Input: lig_pdb (table)

\# Output: update files in lig_h and table lig_initial

\# Prerequisite: set directory to the variable "icmobj_location", "template_location" and "save_location"

$\#-$

\# INPUT VARIABLE

$\mathrm{t}=$ lig_pdb

round = 3 \# number of rounds ( $n$ ) for energy calculation - 3 rounds used in my analysis

\# Input - directory: ensure that your directory to a specific folder ends with "\\"

icmobj_location = "'" \# use save_location specified in the script s2_bicm_conversion

template_location = "" $\quad$ \# use save_location specified in the script s5_lig_h

save_location = "'" \# directory where template table "tt" will be saved

\#-

\#-

function DistCalculation( S )

\#The function calculates and returns the distance before and after adding the methly group \#S=pdb_code//ligand name(protein chain+lig_name)//protein_chain//selected hydrogen atom

$\# S[4]$ is the selected hydrogen atom to be replaced with a methyl group

cp a_\$S[1]. "tmpObj" 
set object a_tmpObj.

$h=a_{-} t m p O \overline{b j} . \$ S[2] / / \$ S[4]$

min_dist1 = Min(Min(Distance $(X y z(h \&$ !a_tmpObj.\$S[2]//vt*), Xyz(a_tmpObj.\$S[3]//*) )))

modifyGroupSmiles $\mathrm{h}$ "C*|1.40,-0.00,0.00,0.00" no no no

min_dist2 $=\operatorname{Min}(\operatorname{Min}(\operatorname{Distance}(\mathrm{Xyz}($ as_graph \& !a_tmpObj.\$S[2]//vt*), Xyz(a_tmpObj.\$S[3] //*) )))

delete a_tmpObj.

delete $\mathrm{S}$

return String(min_dist1)//String(min_dist2)

endfunction

function EnergyDistCalculation( $\mathrm{S}$ )

\#The function calculates and returns the energy vale after replacing a selected hydrogen atom with a methyl group

\#S = pdb_code//ligand name(protein chain+lig_name)//protein_chain//selected hydrogen atom

$\# \mathrm{~S}[4]$ is the selected hydrogen atom to be replaced with a methyl group

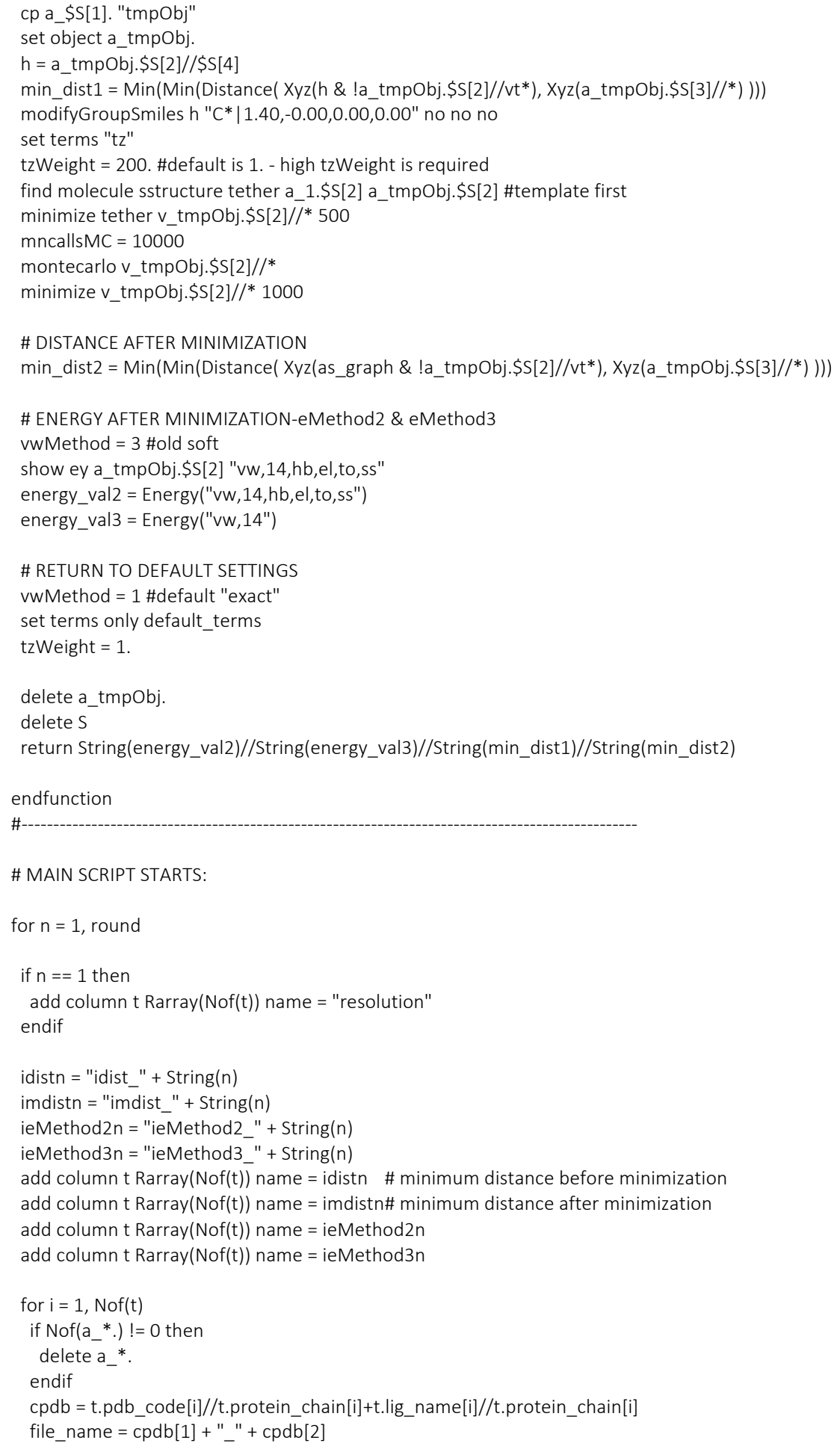


openFile icmobj_location + file_name + ".ob" 0 yes no no no " append" \#icm structure

unds

if $n==1$ then

openFile template_location + file_name + ".icb" \#tt - template table

t.resolution $[\mathrm{i}]=$ Resolution $\left(\mathrm{a} \_\$ c p d b[1]\right.$.)

else

openFile save_location + file_name + ".icb" \#tt - table saved from previous round

endif

\#STEP 1 - calculate distance for each hydrogen atom following modification (no minimization)

distn = "dist_" + String $(n)$

add column tt Rarray(Nof(tt)) name $=$ idistn

add column tt Rarray(Nof(tt)) name $=$ distn

for $\mathrm{a}=1, \operatorname{Nof}(\mathrm{tt})$

$\arg =\mathrm{cpdb} / / \mathrm{tt}$.lig_h[a]

d_result $=$ DistCalculation $(\arg )$

tt.\$idistn[a] = Real(d_result[1])

tt.\$distn[a] = Real(d_result[2])

delete arg, d_result

endfor

\#STEP 2 - calculate initial energy and distance \& record it to $t$ (before and after mnimization)

t.\$idistn[i] = Min(Min(Distance $\left.\left(X y z\left(a \_\$ c p d b[1] . \$ c p d b[2] / / ! v t^{*}\right), X y z\left(a \_\$ c p d b[1] . \$ c p d b[3] / / ! v t^{*}\right)\right)\right)$ )

cp a_\$cpdb[1].\$cpdb[2] "lig_template"

set object a_\$cpdb[1].

set term "tz"

tzWeight $=1$.

find molecule sstructure tether a_lig_template.\$cpdb[2] a_\$cpdb[1].\$cpdb[2] \#template first

minimize $v \$ c p d b[1] . \$ c p d b[2] / / * 1000$

delete a_lig_template.

t.\$imdistn[i] = Min(Min(Distance $\left.\left(X y z\left(a \_\$ c p d b[1] . \$ c p d b[2] / / ! v t^{*}\right), X y z\left(a \_\$ c p d b[1] . \$ c p d b[3] / / ! v t^{*}\right)\right)\right)$ )

vwMethod $=3$ \#old soft

show ey a_\$cpdb[1].\$cpdb[2] "vw,14,hb,el,to,ss"

i_energy2 =Energy("vw,14,hb,el,to,ss")

i_energy3 = Energy("vw,14")

\# return to default settings - omit tzWeight (already default value)

vwMethod = 1 \#default "exact"

set terms only default_terms

t.\$ieMethod2n[i] = Real(i_energy2)

t.\$ieMethod3n[i] = Real(i_energy3)

\# STEP 3 - calculate energy and distance after mini for each hydrogen atom following modification

mdistn = "mdist_" + String $(\mathrm{n})$

eMethod2n = "eMethod2_" + String $(n)$

eMethod3n = "eMethod3_" + String $(n)$

add column tt Rarray(Nof(tt)) name = imdistn

add column tt Rarray(Nof(tt)) name $=$ mdistn

add column tt Rarray(Nof(tt)) name $=$ eMethod $2 \mathrm{n}$

add column tt Rarray (Nof(tt)) name $=$ eMethod $3 \mathrm{n}$

for $\mathrm{a}=1, \operatorname{Nof}(\mathrm{tt})$

$\arg =\mathrm{cpdb} / / \mathrm{tt}$.lig_h[a]

d_result $=$ DistCalculation $(\arg )$

ed_result $=$ EnergyDistCalculation(arg)

tt.\$eMethod2n[a] = Real (ed_result[1])

tt.\$eMethod3n[a] = Real(ed_result[2])

tt.\$imdistn[a] $=$ Real(ed_result[3])

tt.\$mdistn[a] = Real(ed_result[4])

delete arg, ed_result

endfor

\#STEP 4 - save tt and t(update)

I_confirm = no

write binary tt save location + file name + ".icb"

write binary t save_location + "lig_initial.icb"

I_confirm = yes

delete cpdb, file_name, i_energy2, i_energy3, tt

delete eMethod2n, eMethod3n, distn, mdistn

endfor \#for i

delete ieMethod2n, ieMethod3n, idistn, imdistn 
endfor \#for $\mathrm{n}$

lig_initial $=\mathrm{t}$

delete t, round, icmobj_location, template_location, save_location

7- Energy val-AS

errorAction $=3$

\# Purpose: To caclulate average and standard deviation of PDB 3D protein structures' energy values

\# Input: lig_initial (table)

\# Output: Add average and standard deviation values for each tt in pdb and table lig_initial

$\#-$

\# INPUT VARIABLES

$\mathrm{t}=$ lig_initial

round = 3 \# use the identical value as the variable "round" in the script s6_energycal_n

\# Input - directory: ensure that your directory to a specific folder ends with "\\"

table_location = "'" \# use save_location specified in the script s6_energycal_n

\#-

\# MAIN SCRIPT STARTS:

add column $t$ Rarray $(\operatorname{Nof}(t))$ name = "idist_A" \# A stands for average

add column t Rarray(Nof(t)) name = "idist_S"\# S stands for standard deviation

add column t Rarray(Nof(t)) name = "imdist_A"

add column $t$ Rarray(Nof(t)) name = "imdist_S"

add column $t$ Rarray $(\operatorname{Nof}(t))$ name $=$ "ieMethod2 A"

add column t Rarray (Nof(t)) name = "ieMethod2_S"

add column t Rarray(Nof(t)) name = "ieMethod3_A"

add column t Rarray $(\operatorname{Nof}(\mathrm{t}))$ name $=$ "ieMethod3_S"

for $\mathrm{i}=1, \operatorname{Nof}(\mathrm{t})$

$\mathrm{cpdb}=\mathrm{t}$. pdb_code[i]//t.protein_chain[i] + t.lig_name[i]//t.protein chain[i]

file_name $=c p d b[1]+"{ }_{-}+\operatorname{cpdb}[2]$

openFile table_location + file_name + ".icb" \#0 yes no no no "append" \#tt

add column tt Rarray(Nof(tt)) name = "idist A"

add column tt Rarray(Nof(tt)) name = "idist_S"

add column tt Rarray(Nof(tt)) name = "dist_A"

add column tt Rarray(Nof(tt)) name = "dist_S"

add column tt Rarray(Nof(tt)) name = "imdist_A"

add column tt Rarray(Nof(tt)) name = "imdist $\mathrm{S} "$

add column tt Rarray(Nof(tt)) name = "mdist_A"

add column tt Rarray(Nof(tt)) name = "mdist_S"

add column tt Rarray(Nof(tt)) name = "eMethod2_A"

add column tt Rarray(Nof(tt)) name = "eMethod2_S"

add column tt Rarray(Nof(tt)) name = "eMethod3 A"

add column tt Rarray(Nof(tt)) name = "eMethod3_S"

add column tt Rarray(Nof(tt)) name = "deMethod2"

add column tt Rarray(Nof(tt)) name = "deMethod3"

\# STEP 1 - calculate average and sd for initial values: ieMethod2, ieMethod3, and idist

for $n=1$, round

idistn = "idist_" + String(n)

imdistn = "imdist_" + String $(\mathrm{n})$

ieMethod2n = "ieMethod2_" + String(n)

ieMethod3n = "ieMethod3_" + String(n)

if $n==1$ then

idistn_val = Real(t.\$idistn[i])

imdistn_val = Real(t.\$imdistn[i])

ieMethod2n_val = Real(t.\$ieMethod2n[i])

ieMethod3n_val = Real(t.\$ieMethod3n[i])

else

idistn_ov = idistn_val

imdistn_ov = imdistn_val

ieMethod2n_ov = ieMethod2n_val

ieMethod3n_ov = ieMethod3n_val

delete idistn_val, imdistn_val, ieMethod2n_val, ieMethod3n_val

idistn_val = idistn_ov//Real(t.\$idistn[i]) 
imdistn_val = imdistn_ov//Real(t.\$imdistn[i])

ieMethod2n_val = ieMethod2n_ov//Real(t.\$ieMethod2n[i])

ieMethod3n_val = ieMethod3n_ov//Real(t.\$ieMethod3n[i])

delete idistn_ov, imdistn_ov, ieMethod2n_ov, ieMethod3n_ov

endif

delete idistn, imdistn, ieMethod2n, ieMethod3n

endfor \#for $n$

t.idist_A $[i]=$ Sum(idistn_val) $/$ Real(round)

t.idist_S[i] = Rmsd(idistn_val)

t.imdist_A $[i]=$ Sum(imdistn_val)/Real(round)

t.imdist $\mathrm{S}[\mathrm{i}]=\mathrm{Rmsd}(\mathrm{imdistn}$ val)

t.ieMethod2_A[i] = Sum(ieMethod2n_val)/Real $($ round $)$

t.ieMethod2_S[i] = Rmsd(ieMethod2n_val)

t.ieMethod3_A[i] =Sum(ieMethod3n_val)/Real $($ round $)$

t.ieMethod3_S[i] = Rmsd(ieMethod3n_val)

delete idistn_val, imdistn_val, ieMethod2n_val, ieMethod3n_val

\# STEP 2 - calculate average and sd for each row in tt

for $\mathrm{a}=1$, Nof(tt)

idistn = "idist_" + String $(\mathrm{n})$

distn = "dist_" + String(n)

imdistn = "imdist_" + String(n)

mdistn = "mdist_" + String $(\mathrm{n})$

eMethod2n = "eMethod2_" + String(n)

eMethod3n = "eMethod3 " + String $(n)$

if $n==1$ then

idistn_val $=$ Real(tt.\$idistn[a])

distn_val = Real(tt.\$distn[a])

imdistn_val = Real(tt.\$imdistn[a])

mdistn_val = Real(tt.\$mdistn[a])

eMethod2n_val = Real(tt.\$eMethod2n[a])

eMethod3n_val $=$ Real(tt.\$eMethod3n[a])

else

idistn_ov = idistn_val

distn_ov = distn_val

imdistn_ov = imdistn_val

mdistn_ov = mdistn_va

eMethod2n_ov = eMethod2n_val

eMethod3n_ov = eMethod3n_val

delete idistn_val, distn_val, imdistn_val, mdistn_val, eMethod2n_val, eMethod3n_val

idistn_val = idistn_ov//Real(tt.\$idistn[a])

distn_val = distn_ov//Real(tt.\$distn[a])

imdistn_val = imdistn_ov//Real(tt.\$imdistn[a])

mdistn_val = mdistn_ov//Real(tt.\$mdistn[a])

eMethod2n_val = eMethod2n_ov//Real(tt.\$eMethod2n[a])

eMethod3n_val = eMethod3n_ov//Real(tt.\$eMethod3n[a])

delete idistn_ov, distn_ov, imdistn_ov, mdistn_ov, eMethod2n_ov, eMethod3n_ov

endif

delete idistn, distn, imdistn, mdistn, eMethod2n, eMethod3n

endfor \#for $n$

tt.idist_A[a] = Sum(idistn_val)/Real(round)

tt.idist_S[a] $=$ Rmsd(idistn_val)

tt.dist_A[a] = Sum(distn_val)/Real(round)

tt.dist_S[a] = Rmsd(distn_val)

tt.imdist_A[a] = Sum(imdistn_val)/Real(round)

tt.imdist_S[a] $=$ Rmsd(imdistn_val)

tt.mdist_A[a] $=$ Sum(mdistn_val $) /$ Real $($ round $)$

tt.mdist_S[a] $=$ Rmsd(mdistn_val)

tt.eMethod2_A[a] = Sum(eMethod2n_val)/Real(round)

tt.eMethod2_S[a] = Rmsd(eMethod2n_val)

tt.eMethod3_A[a] =Sum(eMethod3n_val)/Real(round)

tt.eMethod3_S[a] = Rmsd(eMethod3n_val)

delete idistn_val, distn_val, imdistn_val, mdistn_val, eMethod2n_val, eMethod3n_val

tt.deMethod2[a]=tt.eMethod2_A[a]-t.ieMethod2_A[i]

tt.deMethod3[a]=tt.eMethod3_A[a]-t.ieMethod3_A[i] 
endfor \#for a

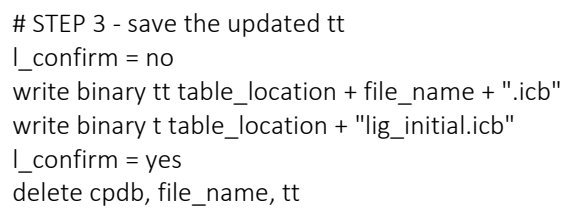

\# Purpose 1: To check the domain names of each 3D protein structure pairs (inserts domain_name1 and domain_name2) \# Purpose 2: If the domain names are identical between two 3D protein structure pairs, the column remove \# $\quad$ will indicate "yes" (refer to NOTE)

\# Input: dlig_rmsdcf (table); ip_distribution_validation (reference table) 
\# Output: dlig_rmsdcf_ip (table)

\# ***NOTE*** - if there are rows to remove, the changes must be updated on the table "dlig_inital" as well.

\# It wasn't necessary for the purpose of this script as there were no rows that was inidicated

\# $\quad$ as "yes" on the column "remove".

\#-

\# INPUT VARIABLES

$\mathrm{t}=$ dlig_rmsdcf

t_ref = ip_distribution_validated

\#-

\# EDIT INPUT TABLE - i.e., ip_distribution_validation table missing some domain names

\# EDIT 1 - add in missing IP IDs to the reference table "t_ref"

add t_ref

t_ref.Domain_ID[Nof(t_ref)]="IPR041667"

t_ref.Domain_Name[Nof(t_ref)]="Cupin-like domain 8"

add_t_ref

t_ref.Domain ID[Nof(t_ref)]="IPR041086"

t_ref.Domain_Name[Nof(t_ref)]="YAP binding domain"

t_ref.Domain_Name[Index(t_ref.Domain_ID "IPR004045")] = "Glutathione S-transferase"

t_ref.Domain_Name[Index(t_ref.Domain_ID "IPR000719")] = "Protein kinase domain"

\# EDIT 2 - replace redundant IP IDs on table "t"

t.ip_code1=Replace(t.ip_code1 "IPR004046" "IPR004045") \#GST

t.ip_code2=Replace(t.ip_code2 "IPR004046" "IPR004045")

t.ip_code1=Replace(t.ip_code1 "IPR001245" "IPR000719") \#Protein kinase domain

t.ip_code2=Replace(t.ip_code2 "IPR001245" "IPR000719")

$\#-$

\# MAIN SCRIPT STARTS:

add column t Sarray(Nof(t)) name="domain name1"

add column t Sarray(Nof(t)) name="domain_name2"

add column t Sarray(Nof(t)) name="remove"

for $i=1, \operatorname{Nof}(t)$

if t.ip_code1[i]==t.ip_code2[i] then

t.remove[i]="yes"

continue

endif

t.domain_name1[i] = t_ref.Domain_Name[Index(t_ref.Domain_ID t.ip_code1[i])]

t.domain_name2[i] = t_ref.Domain_Name[Index(t_ref.Domain_ID t.ip_code2[i])]

endfor

if $\operatorname{Nof}($ t.remove=="yes")==0 then \#NOTE: if not, then write a code to remove it from "dlig_inital" as well delete t.remove

endif

dlig_rmsdcf_ip $=\mathrm{t}$

delete t_ref, $t$

10- H-Match

errorAction=3

\# Purpose 1: To check the domain names of each 3D protein structure pairs (inserts domain_name1 and domain_name2) \# Purpose 2: If the domain names are identical between two 3D protein structure pairs, the column remove \# $\quad$ will indicate "yes" (refer to NOTE)

\# Input: dlig_rmsdcf (table); ip_distribution_validation (reference table)

\# Output: dlig_rmsdcf_ip (table)

\#***NOTE*** - if there are rows to remove, the changes must be updated on the table "dlig inital" as well.

\# It wasn't necessary for the purpose of this script as there were no rows that was inidicated

\# $\quad$ as "yes" on the column "remove".

\# INPUT VARIABLES 
$t=$ dlig_rmsdcf

t_ref $=$ ip_distribution_validated

\#-

\# EDIT INPUT TABLE - i.e., ip distribution validation table missing some domain names

\# EDIT 1 - add in missing IP IDs to the reference table "t_ref"

add t_ref

t_ref.Domain_ID[Nof(t_ref)]="IPR041667"

t_ref.Domain_Name[Nof(t_ref)]="Cupin-like domain 8"

add t ref

t_ref.Domain_ID[Nof(t_ref)]="IPR041086"

t ref.Domain_Name[Nof(t_ref)]="YAP binding domain"

t_ref.Domain_Name[Index(t_ref.Domain_ID "IPR004045")] = "Glutathione S-transferase"

t_ref.Domain_Name[Index(t_ref.Domain_ID "IPR000719")] = "Protein kinase domain"

\# EDIT 2 - replace redundant IP IDs on table "t"

t.ip_code1=Replace(t.ip_code1 "IPR004046" "IPR004045") \#GST

t.ip_code2=Replace(t.ip_code2 "IPR004046" "IPR004045")

t.ip_code1=Replace(t.ip_code1 "IPR001245" "IPR000719") \#Protein kinase domain

t.ip_code2=Replace(t.ip_code2 "IPR001245" "IPR000719")

\# MAIN SCRIPT STARTS:

add column t Sarray $(\operatorname{Nof}(\mathrm{t}))$ name="domain_name1"

add column t Sarray(Nof(t)) name="domain name2"

add column t Sarray(Nof(t)) name="remove"

for $i=1, \operatorname{Nof}(t)$

if t.ip_code1[i]==t.ip_code2[i] then

t.remove[i]="yes"

continue

endif

t.domain_name1[i] = t_ref.Domain_Name[Index(t_ref.Domain_ID t.ip_code1[i])]

t.domain_name2[i] = t_ref.Domain_Name[Index(t_ref.Domain_ID t.ip_code2[i])]

endfor

if Nof(t.remove=="yes")==0 then \#NOTE: if not, then write a code to remove it from "dlig_inital" as well delete t.remove

endif

dlig_rmsdcf_ip $=\mathrm{t}$

delete $t \_r e f, t$

11- Lig-Energy

errorAction $=3$

default_terms = "vw,14,hb,el,to,ss"

\# Purpose: To match hydorgen atom \# (and associated energy values) of ligands between two PDB 3D structures of each pair

\# Input: dlig_rmsdcf_ip (table); dlig_initial (reference table)

\# Output: lig_energy (table)

\# Prerequisite: set directory to the variable "table_location", "h_matched_tt_location" and "save_location"

\# INPUT VARIABLES

$\mathrm{t}=$ dlig_rmsdcf ip

t_ref = dlig_initial

distn = "dist_A" \# depending on the energy column in interest (average vs. single val)

mdistn = "mdist_A"

eMethod2n = "eMethod2 $A$ "

eMethod3n = "eMethod3_A"

deMethod2n = "deMethod2"

deMethod3n = "deMethod3"

\# Input - directory: ensure that your directory to a specific folder ends with "\I" 
table_location = "'" \# use table_location specified in the script s7_energyval_AS

h_matched_tt_location = "" \# use folder location where tables in save_location specified in the script s10_h_match

\# are manually checked and corrected (refer to NOTE below)

save_location = "' \# directory where output table "lig_energy" will be saved

\# NOTE - "h_matched_tt_location" (hydrogen atoms matched template table location) is the location in

\# which template tables of list of hydrogen atoms of the same co-crystalized ligands matched

\# between two PDB structures.

\# IF the hydrogen atoms are matched using built-in function, they are not perfect. Hence, it

\# is required to be manually checked as also written in the script s10_h_match.

\section{\# MAIN SCRIPT STARTS:}

add column t Sarray(Nof(t)) name = "error_s7"

if Nof(a_*.) != 0 then

delete $a_{-}^{*}$.

endif

for $\mathrm{i}=1, \operatorname{Nof}(\mathrm{t})$

index1=Index(t_ref.pdb_code t.pdb1[i])

index2=Index(t_ref.pdb_code t.pdb2[i])

pdb1=t.pdb1[i]//t_ref.protein_chain[index1]+t.lig_name[i]//t.lig_name[i]

pdb2=t.pdb2[i]//t_ref.protein_chain[index2]+t.lig_name[i]//t.lig_name[i]

delete index1,index 2

file name1 $=$ pdb1[1] + " " + pdb1[2]

file_name $2=$ pdb2[1] + "- + pdb2[2]

file_name $=\operatorname{pdb} 1[2]+$ "_" pdb1[1] + "_" + pdb2[1]

openFile table_location + file_name1 + ".icb" \#tt rename tt Name(Name("pdb1t" simple),unique) openFile table_location + file_name2 + ".icb" \#tt rename tt Name(Name("pdb2t" simple), unique) if Nof(pdb1t) != Nof(pdb2t) then \#error check delete index1, index2, pdb1, pdb2, pdb1t, pdb2t t.error_s7[i] = "yes" continue endif

openFile h_matched_tt_location + file_name + ".icb" \#tt - matched h table

\#STEP 2 - organize information based on mathcing hydrogens (tt) into lig_energy add header lig_energy "A new table" name = "title" add column lig_energy Sarray(0) name = "pdb1_h" add column lig_energy $\operatorname{Sarray}(0)$ name $=$ "pdb2_h" add column lig_energy Sarray(0) name = "methyl_h" add column lig_energy Rarray(0) name = "pdb1_dist" add column lig_energy Rarray(0) name = "pdb2_dist" add column lig_energy Rarray(0) name = "pdb1_mdist" add column lig_energy Rarray(0) name = "pdb2_mdist" add column lig_energy Rarray(0) name = "pdb1_eMethod2" add column lig_energy $\operatorname{Rarray}(0)$ name = "pdb2_eMethod2" add column lig_energy Rarray $(0)$ name = "pdb1_eMethod3" add column lig_energy $\operatorname{Rarray}(0)$ name = "pdb2_eMethod3"

$\mathrm{a}=1$

sort(pdb1t.bound_atom)

while $a<\operatorname{Nof}(p d b 1 t)+1$ \#while $h$ is less than number of hydrogen atoms

add lig_energy

if pdb1t.methyl[a] == "yes" then \#if methyl then find and use the lowest energy val lig_energy.methyl_h[Nof(lig_energy)] = "yes"

t_temp1 = pdb1t.bound_atom $==$ pdb1t.bound_atom[a]

t_temp2 = pdb2t.bound_atom == tt.pdb2_atom[Index(tt.pdb1_atom pdb1t.bound_atom[a])]

pdb1_h = t_temp1.lig_h[1] + "/" + t_temp1.lig_h[2] + "/" + t_temp1.lig_h[3]

h1_index = Index(t_temp2.lig_h tt.pdb2_atom[Index(tt.pdb1_atom t_temp1.lig_h[1])])

h2_index $=$ Index(t_temp2.lig_h tt.pdb2_atom[Index(tt.pdb1_atom t_temp1.lig_h[2])])

h3_index = Index(t_temp2.lig_h tt.pdb2_atom[Index(tt.pdb1_atom t_temp1.lig_h[3])])

pdb2_h = t_temp2.lig_h[h1_index] + "/" + t_temp2.lig_h[h2_index] + "/" + t_temp2.lig_h[h3_index] 
highest_dist = Sort(t_temp1.\$distn) [Nof(t_temp1)]//Sort(t_temp2.\$distn)[Nof(t_temp2)]

highest_mdist = Sort(t_temp1.\$mdistn)[Nof(t_temp1)]//Sort(t_temp2.\$mdistn)[Nof(t_temp2)]

lowest_eMethod2 = Sort(t_temp1.\$eMethod2n)[1]//Sort(t_temp2.\$eMethod2n)[1]

lowest_eMethod3 = Sort(t_temp1.\$eMethod3n)[1]//Sort(t_temp2.\$eMethod3n)[1]

pdb1_info $=$ highest_dist[1]//highest_mdist[1]//lowest_eMethod2[1]//lowest_eMethod3[1]

pdb2_info = highest_dist[2]//highest_mdist[2]//lowest_eMethod2[2]//lowest_eMethod3[2]

$\mathrm{a}=\mathrm{a}+3$

delete t_temp1, t_temp2, h1_index, h2_index, h3_index

delete highest_dist, highest_mdist, lowest_eMethod2, lowest_eMethod3

else

pdb1_h = pdb1t.lig_h[a]

pdb1_info = pdb1t.\$distn[a]//pdb1t.\$mdistn[a]//pdb1t.\$eMethod2n[a]//pdb1t.\$eMethod3n[a]

pdb2_h = tt.pdb2_atom[Index(tt.pdb1_atom pdb1_h)]

pdb2_index = Index (pdb2t.lig_h pdb2_h)

pdb2_info = pdb2t.\$distn[pdb2_index]//pdb2t.\$mdistn[pdb2_index]//pdb2t.\$eMethod2n[pdb2_index]//pdb2t.\$eMethod3n[pdb2_index]

$a=a+1$

delete pdb2_index

endif

lig_energy.pdb1_h[Nof(lig_energy)] $=p d b 1 \_h$

lig_energy.pdb2_h[Nof(lig_energy)] $=$ pdb2_h

lig_energy.pdb1_dist[Nof(lig_energy)] = pdb1_info[1]

lig_energy.pdb2_dist[Nof(lig_energy)] = pdb2_info[1]

lig_energy.pdb1_mdist[Nof(lig_energy)] = pdb1_info[2]

lig_energy.pdb2_mdist[Nof(lig_energy)] = pdb2_info[2]

lig_energy.pdb1_eMethod2[Nof(lig_energy)] = pdb1_info[3]

lig_energy.pdb2 eMethod2[Nof(lig_energy)] = pdb2 info[3]

lig_energy.pdb1_eMethod3[Nof(lig_energy)] = pdb1_info[4]

lig_energy.pdb2_eMethod3[Nof(lig_energy)] = pdb2_info[4]

delete pdb1_h, pdb2_h, pdb1_info, pdb2_info

endwhile

\#STEP 3 - save lig_energy

I_confirm = no

write binary lig_energy save_location + file_name + ".icb"

I_confirm = yes

delete $p d b 1, p d b 2$, pdb1t, pdb2t, h_match, file_name1, file_name2, file_name, tt, lig_energy

endfor

if Nof(t.error_s7 == "yes") != 0 then

error $s 7=t$

endif

delete $t, t$ _ref, distn, mdistn, eMethod2n, eMethod3n, save_location

12- Percent risk

errorAction $=3$

\# Purpose: To calculate percent risk, pcR (previously termed shared percentage (sp)).

\# $\quad$ "lig_energy" tables generated and saved in the script s12_lig_energy are updated.

\# Input: dlig_rmsdcf_ip (table); dlig_initial (reference table)

\# Output: lig_sp

\# Prerequisite: set directory to the variable "table_location" and "save_location"

\# INPUT VARIABLES

$\mathrm{t}=$ dlig_rmsdcf_ip

t_ref = dlig_initial

\# Input - directory: ensure that your directory to a specific folder ends with "\\"

table_location = "'" \# use save_location specified in the script s11_lig_energy

save_location = "'" \# directory where updated "lig_energy" tables will be saved

dist_threshold=1.6 \# threshold/cut-off values

eMethod2_threshold $=0$.

eMethod3_threshold $=0$.

\#- 


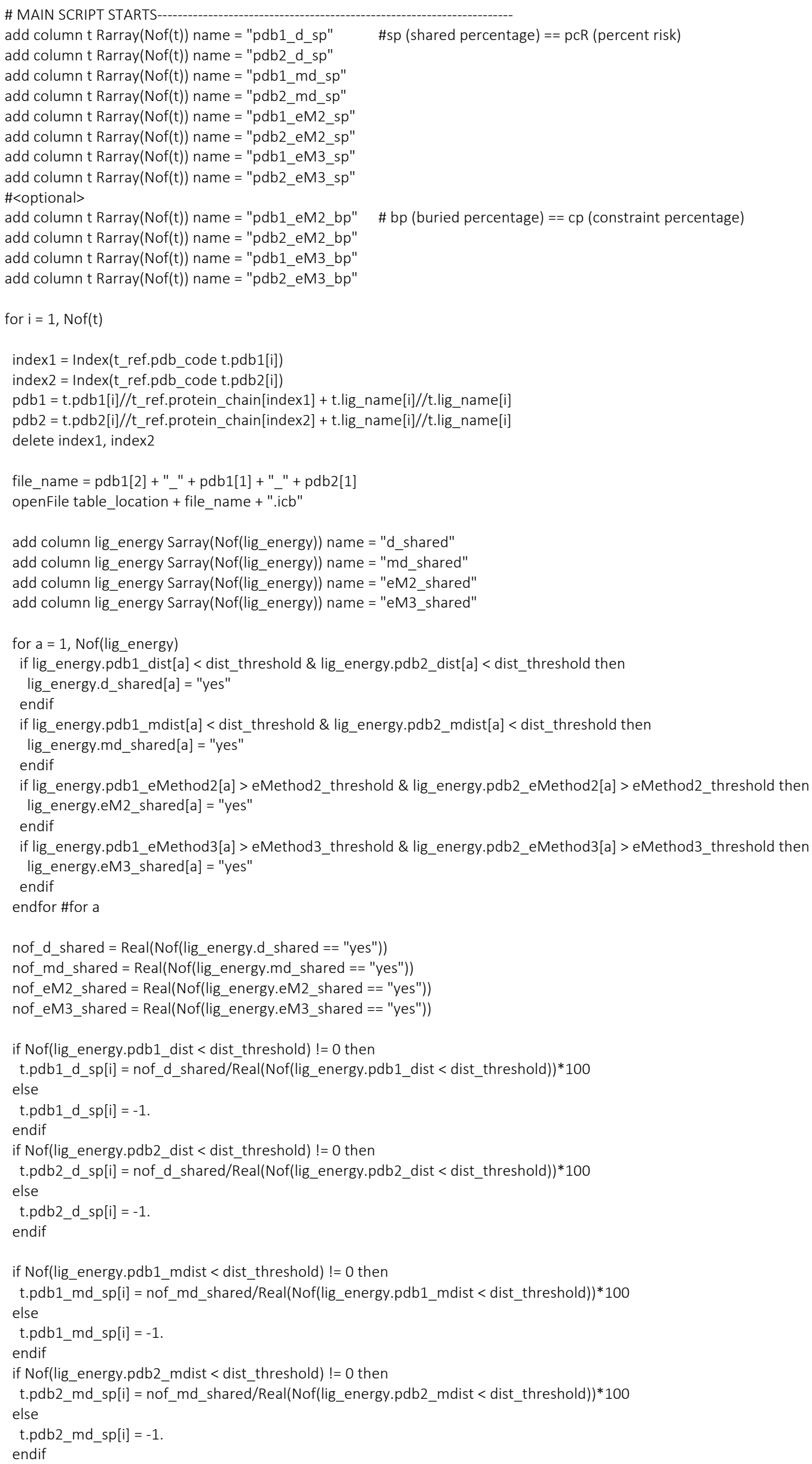


if Nof(lig_energy.pdb1_eMethod2 > eMethod2_threshold) != 0 then

t.pdb1_eM2_sp[i] = nof_eM2_shared/Real(Nof(lig_energy.pdb1_eMethod2 > eMethod2_threshold))*100

t.pdb1_eM2_bp[i] = Real(Nof(lig_energy.pdb1_eMethod2 > eMethod2_threshold))/Real(Nof(lig_energy))*100

else

t.pdb1_eM2_sp[i] $=-1$.

t.pdb1 eM2 bp $[i]=0$.

endif

if Nof(lig_energy.pdb2_eMethod2 > eMethod2_threshold) != 0 then

t.pdb2_eM2_sp[i] = nof_eM2_shared/Real(Nof(lig_energy.pdb2_eMethod2 > eMethod2_threshold))*100

t.pdb2_eM2_bp[i] = Real(Nof(lig_energy.pdb2_eMethod2>eMethod2_threshold))/Real(Nof(lig_energy))*100

else

t.pdb2_eM2_sp[i] $=-1$.

t.pdb2_eM2_bp $[i]=0$.

endif

if Nof(lig_energy.pdb1 eMethod3 > eMethod3 threshold) != 0 then

t.pdb1_eM3_sp[i] = nof_eM3_shared/Real(Nof(lig_energy.pdb1_eMethod3 > eMethod3_threshold))*100

t.pdb1_eM3_bp[i] = Real(Nof(lig_energy.pdb1_eMethod3 > eMethod3_threshold))/Real(Nof(lig_energy))*100

else

t.pdb1_eM3_sp[i] $=-1$.

t.pdb1_eM3_sp[i] $=0$.

endif

if Nof(lig_energy.pdb2_eMethod3 > eMethod3 threshold) != 0 then

t.pdb2_eM3_sp[i] = nof_eM3_shared/Real(Nof(lig_energy.pdb2_eMethod3 > eMethod3_threshold))*100

t.pdb2_eM3_bp[i] = Real(Nof(lig_energy.pdb2_eMethod3 > eMethod3_threshold))/Real(Nof(lig_energy))*100

else

t.pdb2_eM3_sp[i] $=-1$.

t.pdb2_eM3_bp[i] = 0 .

endif

delete nof_d_shared, nof_md_shared, nof_eM2_shared, nof_eM3_shared

I confirm = no

write binary lig_energy save_location + file_name + ".icb"

write binary t save_location + "lig_sp.icb"

I_confirm =yes

delete pdb1, pdb2, file_name, lig_energy

endfor

lig_sp $=\mathrm{t}$

delete t, t_ref, dist_threshold, eMethod2_threshold, eMethod3_threshold

delete save_location, table_location 


\section{References:}

1. Wang, J., Yazdani, S., Han, A. \& Schapira, M. Structure-based view of the druggable genome. Drug Discov Today 25, 561-567 (2020).

2. Structural Genomics Consortium. NVS-BPTF-1 A chemical probe for BPTF. https://www.thesgc.org/chemical-probes/NVS-BPTF1.

3. Structural Genomics Consortium. NVS-MLLT-1 A Potent and Selective inhibitor of YEATS proteins. https://www.thesgc.org/chemical-probes/NVS-MLLT-1.

4. Bromberg, K. D. et al. The SUV4-20 inhibitor A-196 verifies a role for epigenetics in genomic integrity. Nat. Chem. Biol. 13, 317324 (2017).

5. Structural Genomics Consortium. LLY-507 A chemical probe for SMYD2 protein lysine methyltransferase. https://www.thesgc.org/chemical-probes/LLY-507.

6. Forster, M. et al. Selective JAK3 Inhibitors with a Covalent Reversible Binding Mode Targeting a New Induced Fit Binding Pocket. Cell Chemical Biology 23, 1335-1340 (2016).

7. Structural Genomics Consortium. FM-381 A Chemical Probe For JAK3. https://www.thesgc.org/chemical-probes/FM-381.

8. Rahm, F. et al. Creation of a Novel Class of Potent and Selective MutT Homologue 1 (MTH1) Inhibitors Using Fragment-Based Screening and Structure-Based Drug Design. J. Med. Chem. 61, 2533-2551 (2018).

9. Wilson, J. E. et al. Discovery of CPI-1612: A Potent, Selective, and Orally Bioavailable EP300/CBP Histone Acetyltransferase Inhibitor. ACS Med. Chem. Lett. 11, 1324-1329 (2020). 